\title{
Event-Based Time Varying Formation Control for Multiple Quadrotor UAVs with Markovian Switching Topologies
}

\author{
Zhen Zhou (D), Hongbin Wang (D), and Zhongquan Hu \\ Key Lab of Industrial Computer Control Engineering of Hebei Province, Yanshan University, Qinhuangdao 066004, China \\ Correspondence should be addressed to Zhen Zhou; zhouzhen0617@163.com
}

Received 22 December 2017; Accepted 20 February 2018; Published 23 April 2018

Academic Editor: Victor M. Becerra

Copyright (c) 2018 Zhen Zhou et al. This is an open access article distributed under the Creative Commons Attribution License, which permits unrestricted use, distribution, and reproduction in any medium, provided the original work is properly cited.

\begin{abstract}
Time varying formation control problem for a group of quadrotor unmanned aerial vehicles (UAVs) under Markovian switching topologies is investigated through a modified dynamic event-triggered control protocol. The formation shape is specified by a time varying vector, which prescribes the relative positions and bearings among the whole agents. Instead of the general stochastic topology, the graph is governed by a set of Markov chains to the edges, which can recover the traditional Markovian switching topologies in line with the practical communication network. The stability proof for the state space origin of the overall closedloop system is derived from the singular perturbation method and Lyapunov stability theory. An event-triggered formation control protocol in terms of a dynamically varying threshold parameter is delicately carried out, while acquiring satisfactory resource efficiency, and Zeno behavior of triggering time sequences is excluded. Finally, simulations on six quadrotor UAVs are given to verify the effectiveness of the theoretical results.
\end{abstract}

\section{Introduction}

Along with the increasing applications in various areas, such as aerial photography, express delivery, and disaster relief, formation control of multiagent systems has attracted considerable attention from many researchers $[1,2]$. In particular, as a typical class of physical systems with practical interest, the quadrotor UAVs is widely used in the military and civilian fields $[3,4]$. Actually, due to the strong nonlinear coupling and limited communication resources [5], the control problem of multiple quadrotor UAVs will be very challenging and difficult [6]. Therefore, how to design the formation control protocol for multiple quadrotor UAVs subject to limited communication resources becomes a significant research focus.

A defining feature of formation control problem is that multiple agents work together to accomplish a collaborative formation task [7]. Several classic formation control strategies, including leader-follower, virtual structure, and behavior based methods, were applied in the scientific community $[8,9]$. For example, formation control of multiple quadrotor UAVs, based on position estimation [10], backstepping design technique [11], and finite time algorithms [12], respectively, was investigated so as to make a construct and keep the formation shape during flying. It should be pointed out that time varying formation tracking problems arise in some scenarios, such as source seeking and target enclosing. For example, time varying formation analysis and design problems for multiagent systems with switching topologies were solved in $[13,14]$. Based on the fact that multiagent systems subject to random abrupt variations could be modeled as the switching systems, then some results have been obtained on it [14]. Compared with the previous works, time varying formation control results for multiagent systems with switching topologies were provided in $[15,16]$. Besides, due to random link failures, variation meeting the need and sudden environmental disturbances [17], some dynamical systems could be modeled as Markovian switching systems, which were governed by a set of Markov chains [18, 19]. By considering the complex network as Markovian switching topologies, it plays a crucial role in the field of networked control system [20].

In practice, under a limited bandwidth, it is necessary and important to consider the issues of energy waste and competition [21]. Therefore, event-triggered communication mechanism was born at the right moment [22, 23]. As a popular research topic, some latest event-triggered control 
results were provided in [24-28]. In particular, compared with the general event-triggered controller with a fixed threshold parameter, the authors in $[24,29]$ developed the dynamic/adaptive event-triggered control protocol of multiagent systems for acquiring satisfactory resource efficiency, respectively. Meanwhile, take the strong nonlinear coupling and underactuated of the quadrotor UAVs into account, time scaling based control method has also been recognized as a powerful tool in the analysis and design of controllers, which is with crucial importance in applications to the mobile inverted pendulum [30], the ball-beam system [31], and the quadrotor UAVs [32]. Therefore, it is of great importance to extend the event-triggered formation results to multiple quadrotor UAVs under Markovian switching topologies. In addition, it is difficult to obtain all the transition rates under the realistic communication environment [33]. So that randomly occurring control strategy is more realistic and meaningful to accomplish attitude stabilization and formation missions under the limited communication resources.

Motivated by these observations, the contributions of this paper are proposing a novel time varying formation control strategy and an event-triggered communication scheme to solve the formation problem of multiple quadrotor UAVs with Markovian switching topologies. The main highlights of this paper are summarized as follows. First, a modified graph of entire system is governed by a set of Markov chains to the edges, and the traditional Markovian switching topologies can be recovered through adjusting the modes of edges and the transition rates. Second, the dynamic eventtriggered controller is derived from a time scaling based control strategy, which consists of two parts: the closed-loop system stability analysis based on the framework of singularly perturbed theory and the event-triggered control scheme in terms of a new dynamically varying threshold parameter to guarantee time varying formation shape. Third, Markovian switching topologies involve partly unknown transition rates, which are of great importance to be considered and thus closer to the realistic communication environment. In addition, Zeno behavior can be excluded during the whole running process. Finally, several simulations can illustrate the theoretical results.

The rest of this paper is organized as follows. The system dynamics and some preliminaries on graph theory are introduced in Section 2; Section 3 provides main results on event-triggered formation control for multiple quadrotor UAVs. In Section 4, simulation results are given and this paper is concluded in Section 5 .

Notations 1. Throughout this paper, $\|\cdot\|$ denotes $L_{2}$-vector norm and $\rho(\bullet)$ stands for the spectral radius for matrices. The notation $A \otimes B$ means the Kronecker product of matrices $A$ and $B$, and $\lambda_{\text {max }}(\bullet)$ and $\lambda_{\text {min }}(\bullet)$ represent its maximum and minimum eigenvalues.

\section{Preliminaries and System Formulation}

2.1. Graph Theory. Define a time varying random undirected graph $G(\omega(t))=\left(\nu, \xi\left(\omega_{i j}(t)\right), A(\omega(t))\right)$ with a nonempty finite vertex set $v=\left\{v_{1}, \ldots, \nu_{N}\right\}$ and an edge set $\xi\left(\omega_{i j}(t)\right) \subseteq \nu \times \nu$.
Different with the general ones, it consists of a time sequence of random graphs in which the edge set $\xi$ varies with $t$. Namely, each edge $\left(v_{i}, v_{j}\right)$ evolves according to a homogeneous Markov process $\omega_{i j}(t)$, which takes values in $S=$ $\{1,2, \ldots, s\}$ with the transition rate as

$$
\begin{gathered}
\operatorname{Pr}\left\{\omega_{i j}(t+\Delta)=q \mid \omega_{i j}(t)=p\right\} \\
= \begin{cases}\pi_{p q} \Delta+o(\Delta) & q \neq p \\
1+\pi_{p p} \Delta+o(\Delta) & q=p .\end{cases}
\end{gathered}
$$

Assume that $\omega_{i j}(t)$ do not change infinitely fast; thus, $\omega_{i j}(t)=$ $\omega_{i j}\left(t+\Delta_{t}\right)$ if $0<\Delta_{t}<\Delta$. It means that the total number of system modes is $s^{(N+1) N / 2}$ and the total transition rate is given by

$$
\begin{aligned}
\operatorname{Pr} & \{\omega(t+\Delta) \mid \omega(t)\} \\
& =\prod_{i, j \in \nu} \operatorname{Pr}\left\{\omega_{i j}(t+\Delta)=q \mid \omega_{i j}(t)=p\right\} .
\end{aligned}
$$

The weighted adjacency matrix $A^{\omega} \triangleq A(\omega(t))=$ $\left[a_{i j}\left(\omega_{i j}(t)\right)\right] \in \mathbb{R}^{N \times N}$ is associated with $G(\omega(t))$. Here $a_{i j}^{\omega} \triangleq$ $a_{i j}\left(\omega_{i j}(t)\right)>0$ if $\left(v_{i}, v_{j}\right) \in \xi\left(\omega_{i j}(t)\right)$ and $a_{i j}^{\omega}=0$ otherwise. Assumed that there is no self-loop in the graph, which implies that $a_{i i}^{\omega}=0$. In this paper, the set of neighbors with respect to the agent $v_{i}$ is $\Omega_{i}^{\omega}=\left\{v_{j} \in v \mid\left(\nu_{j}, v_{i}\right) \in \xi\left(\omega_{i j}(t)\right)\right\}$. A graph $G(\omega(t))$ is connected, if there is a path between any two vertices; otherwise, it is disconnected. A diagonal matrix $D^{\omega}=\operatorname{diag}\left\{d_{i}\right\} \in \mathbb{R}^{N \times N}$ with $d_{i}=\sum_{j \in \Omega_{i}^{\omega}} a_{i j}^{\omega}$ being the $i$ th row sum of $A^{\omega}$. Then, the Laplacian of the graph is defined as $L^{\omega}=D^{\omega}-A^{\omega}$. Consider the formation with a leader-follower structure by introducing a diagonal matrix $L^{l}=\operatorname{diag}\left\{a_{i i}^{l}\right\} \in$ $\mathbb{R}^{N \times N}$, which evolves according to the Markov process $\omega_{0}(t)$ with a finite mode set $S_{0}=\left\{1,2, \ldots, s_{0}\right\}$ and a time interval $\Delta_{0}$, where $a_{i i}^{l}>0$ if $v_{i}$ is a leader and $a_{i i}^{l}=0$ otherwise. Hence, the interaction matrix is given by $L^{G}=L^{\omega}+L^{l}$.

Assumption 2. The undirected graph $G(\omega(t))$ is connected.

Remark 3. For simplicity, we just consider the undirected graph in this paper, that is, $a_{i j}^{\omega}=a_{j i}^{\omega}$. Note that, if the graph is a general directed one, there will be some small differences. A possible approach to consider a directed graph is to introduce two Markov chains in each two agents; an alternative is to extend the state space set $S$, which could be defined according to the weight and direction of the graph. Both approaches will be addressed in our future work.

Remark 4. With a limited bandwidth, the switching of $a_{i j}^{\omega}$ is caused by the sensing/detecting failure and communication failure, which is passive. In fact, it is difficult to obtain all the elements of the transition rate matrix, or some of the elements are not necessary to guarantee the system stability. Since that, the transition rate matrices are assumed to be partly accessed; 

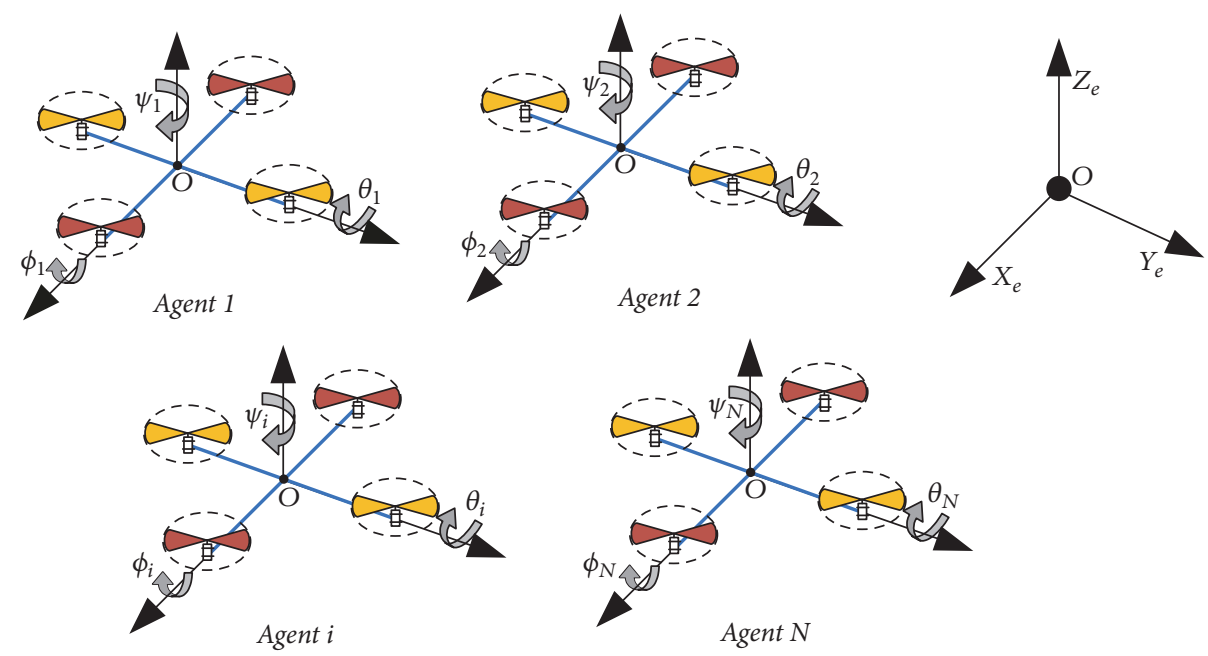

FIGURE 1: Group of the $N$ quadrotor UAVs.

even some of them are unknown completely, which could be descried as follows:

$$
\begin{gathered}
\left(\begin{array}{cccc}
p_{11} & p_{12} & \cdots & ? \\
? & ? & \cdots & p_{2 s} \\
\vdots & \vdots & \ddots & \vdots \\
p_{s 1} & ? & \cdots & p_{s s}
\end{array}\right) \\
\left(\begin{array}{cccc}
? & ? & \cdots & ? \\
? & ? & \cdots & ? \\
\vdots & \vdots & \ddots & \vdots \\
? & ? & \cdots & ?
\end{array}\right)
\end{gathered}
$$

where "?" represents the unknown transition rate.

2.2. Problem Formulation. Consider a group of $N$ quadrotor UAVs as shown in Figure 1; the dynamics of agent $i$ are given as the following form [12]:

$$
\begin{aligned}
& \ddot{x}_{i}=\frac{\left(\sin \psi_{i} \sin \phi_{i}+\cos \psi_{i} \sin \theta_{i} \cos \phi_{i}\right) u_{1 i}}{m_{i}}-\frac{k_{x i} \dot{x}_{i}}{m_{i}}, \\
& \ddot{y}_{i}=\frac{\left(-\cos \psi_{i} \sin \phi_{i}+\sin \psi_{i} \sin \theta_{i} \cos \phi_{i}\right) u_{1 i}}{m_{i}}-\frac{k_{y i} \dot{y}_{i}}{m_{i}}, \\
& \ddot{z}_{i}=-g+\frac{\left(\cos \theta_{i} \cos \phi_{i}\right) u_{1 i}}{m_{i}}-\frac{k_{z i} \dot{z}_{i}}{m_{i}}, \\
& \ddot{\phi}_{i}=\frac{l_{i} u_{2 i}}{I_{x i}}-\frac{l_{i} k_{\phi i} \dot{\phi}_{i}}{I_{x i}}, \\
& \ddot{\theta}_{i}=\frac{l_{i} u_{3 i}}{I_{y i}}-\frac{l_{i} k_{\theta i} \dot{\theta}_{i}}{I_{y i}}, \\
& \ddot{\psi}_{i}=\frac{K_{\psi i} u_{4 i}}{I_{z i}}-\frac{k_{\psi i} \dot{\psi}_{i}}{I_{z i}}
\end{aligned}
$$

where $x_{i}, y_{i}, z_{i} \in \mathbb{R}$ are positions and $\phi_{i}, \theta_{i}, \psi_{i} \in(-\pi / 2, \pi / 2)$ denote the three Euler angles of rotation, representing pitch, roll, and yaw, respectively. $k_{\phi i, \theta i, \psi i}$ are the aerodynamic friction coefficients, and $k_{x i, y i, z i}$ are the coefficients of the translation drag forces. $I_{x i, y i, z i}$ are the quadrotor moments of inertias, $m_{i}$ denotes the mass of the quadrotor, $l_{i}$ is half length of the helicopter, $K_{\psi i}$ is thrust to moment gain, and $g$ is gravitational acceleration.

Each system dynamics consist of six degrees of freedom model, which can be separated into position dynamic and attitude dynamic. With the choice of state variables $x_{1 i}=x_{i}$, $x_{2 i}=\dot{x}_{i} ; y_{1 i}=y_{i}, y_{2 i}=\dot{y}_{i} ; z_{1 i}=z_{i}, z_{2 i}=\dot{z}_{i} ; \phi_{1 i}=\phi_{i}$, $\phi_{2 i}=\dot{\phi}_{i} ; \theta_{1 i}=\theta_{i}, \theta_{2 i}=\dot{\theta}_{i} ; \psi_{1 i}=\psi_{i}, \psi_{2 i}=\dot{\psi}_{i}$. Then, it can be represented by

$$
\begin{aligned}
\dot{x}_{1 i}= & x_{2 i}, \\
\dot{y}_{1 i}= & y_{2 i}, \\
\dot{z}_{1 i}= & z_{2 i}, \\
\dot{x}_{2 i}= & \frac{\left(\sin \psi_{i} \sin \phi_{i}+\cos \psi_{i} \sin \theta_{i} \cos \phi_{i}\right) u_{1 i}}{m_{i}}-\frac{k_{x i} x_{2 i}}{m_{i}}, \\
\dot{y}_{2 i}= & \frac{\left(-\cos \psi_{i} \sin \phi_{i}+\sin \psi_{i} \sin \theta_{i} \cos \phi_{i}\right) u_{1 i}}{m_{i}} \\
& -\frac{k_{y i} y_{2 i}}{m_{i}}, \\
\dot{z}_{2 i}= & -g+\frac{\left(\cos \theta_{i} \cos \phi_{i}\right) u_{1 i}}{m_{i}}-\frac{k_{z i} z_{2 i}}{m_{i}}, \\
\dot{\phi}_{1 i}= & \phi_{2 i}, \\
\dot{\theta}_{1 i}= & \theta_{2 i}, \\
\dot{\psi}_{1 i}= & \psi_{2 i}, \\
\dot{\phi}_{2 i}= & \frac{l_{i} u_{2 i}}{I_{x i}}-\frac{l_{i} k_{\phi i} \phi_{2 i}}{I_{x i}},
\end{aligned}
$$




$$
\begin{gathered}
\dot{\theta}_{2 i}=\frac{l_{i} u_{3 i}}{I_{y i}}-\frac{l_{i} k_{\theta i} \theta_{2 i}}{I_{y i}}, \\
\dot{\psi}_{2 i}=\frac{K_{\psi i} u_{4 i}}{I_{z i}}-\frac{k_{\psi i} \psi_{2 i}}{I_{z i}} .
\end{gathered}
$$

As is well known to us, the quadrotor is underactuated and differentially flat. Accordingly, choose four variables and specify the desired trajectory as $R_{d}=$ $\left[x_{d}(t), y_{d}(t), z_{d}(t), \psi_{d}(t)\right]^{T}$.

2.3. Formation Definition. The formation shape can be described by a vector $\delta(t)=\left[\delta_{1}^{T}(t), \ldots, \delta_{N}^{T}(t)\right]^{T} \in \mathbb{R}^{4 N}$, where $\delta_{i}(t)=\left[\delta_{i x}(t), \delta_{i y}(t), \delta_{i z}(t), \delta_{i \psi}(t)\right]^{T}$ is the continuously differentiable formation vector, such that

$$
\begin{aligned}
& \lim _{t \rightarrow \infty}\left(x_{1 i}(t)-\delta_{i x}(t)-x_{d}(t)\right)=0, \\
& \lim _{t \rightarrow \infty}\left(y_{1 i}(t)-\delta_{i y}(t)-y_{d}(t)\right)=0, \\
& \lim _{t \rightarrow \infty}\left(z_{1 i}(t)-\delta_{i z}(t)-z_{d}(t)\right)=0, \\
& \lim _{t \rightarrow \infty}\left(\psi_{1 i}(t)-\delta_{i \psi}(t)-\psi_{d}(t)\right)=0 .
\end{aligned}
$$

Define the desired position of agent $i$ as $\left[x_{i d}, y_{i d}, z_{i d}, \psi_{i d}\right]^{T}=$ $\left[\delta_{i x}+x_{d}, \delta_{i y}+y_{d}, \delta_{i z}+z_{d}, \delta_{i \psi}+\psi_{d}\right]^{T}$. For $j \in \Omega_{i}^{\omega}$, there exists $\delta_{i j}=\left[\delta_{i j x}, \delta_{i j y}, \delta_{i j z}, \delta_{i j \psi}\right]^{T}=\left[\delta_{j x}-\delta_{i x}, \delta_{j y}-\delta_{i y}, \delta_{j z}-\right.$ $\left.\delta_{i z}, \delta_{j \psi}-\delta_{i \psi}\right]^{T}$. Hence, there are $N(N-1) / 2$ such shape vectors satisfying the following properties:

$$
\begin{aligned}
\delta_{i k} & =\delta_{i j}+\delta_{j k}, \\
\delta_{i i} & =[0,0,0,0]^{T}, \\
\delta_{j i} & =-\delta_{i j},
\end{aligned}
$$

$$
\forall i, j, k \in 1, \ldots, N \text {. }
$$

Remark 5. The formation reference vector $R_{d}$ is not available to all agents, but the desired interdistances of agent $i$ and its neighbors are known. Note that the time varying formation vector $\delta(t)$ is not unique, and the relative position between the reference vector $R_{d}$ and the formation can be adjusted.

Lemma 6 (see [34]). For any $X, Y \in \mathbb{R}^{n}$ and $\rho_{1}>0$, it holds that $X^{T} Y \leq \rho_{1} X^{T} X / 2+Y^{T} Y / 2 \rho_{1}$.

\section{Main Results}

In this section, time varying formation control problem for multiple quadrotor UAVs is solved through an eventtriggered control scheme. The system stability analysis and exclusion of Zeno behavior are also provided.

3.1. Singularly Perturbed System. Define the error vectors as

$$
\begin{aligned}
\sigma_{1}^{2} e_{1 \phi}^{i} & =\phi_{1 i}-\phi_{i d}, \\
\sigma_{1} e_{2 \phi}^{i} & =\phi_{2 i}-\dot{\phi}_{i d}, \\
\sigma_{1}^{2} e_{1 \theta}^{i} & =\theta_{1 i}-\theta_{i d}, \\
\sigma_{1} e_{2 \theta}^{i} & =\theta_{2 i}-\dot{\theta}_{i d}, \\
\sigma_{1}^{2} \widetilde{\psi}_{1 i} & =\psi_{1 i}-\psi_{i d}, \\
\sigma_{1} \widetilde{\psi}_{2 i} & =\psi_{2 i}-\dot{\psi}_{i d}, \\
\tilde{x}_{1 i} & =x_{1 i}-x_{i d}, \\
\tilde{x}_{2 i} & =x_{2 i}-\dot{x}_{i d}, \\
\tilde{y}_{1 i} & =y_{1 i}-y_{i d}, \\
\tilde{y}_{2 i} & =y_{2 i}-\dot{y}_{i d}, \\
\tilde{z}_{1 i} & =z_{1 i}-z_{i d}, \\
\tilde{z}_{2 i} & =z_{2 i}-\dot{z}_{i d},
\end{aligned}
$$

where $\sigma_{1}$ is positive constant denoted as perturbing parameter, which satisfies $\sigma_{1} \ll 1$. The overall system can be written as

$$
\begin{aligned}
& \sum_{\phi i}:=\left\{\begin{array}{l}
\sigma_{1} \dot{e}_{1 \phi}^{i}=e_{2 \phi}^{i} \\
\sigma_{1} \dot{e}_{2 \phi}^{i}=\frac{l_{i} u_{2 i}}{I_{x i}}-\frac{l_{i} k_{\phi i} \phi_{2 i}}{I_{x i}}-\ddot{\phi}_{i d},
\end{array}\right. \\
& \sum_{\theta i}:=\left\{\begin{array}{l}
\sigma_{1} \dot{e}_{1 \theta}^{i}=e_{2 \theta}^{i} \\
\sigma_{1} \dot{e}_{2 \theta}^{i}=\frac{l_{i} u_{3 i}}{I_{y i}}-\frac{l_{i} k_{\theta i} \theta_{2 i}}{I_{y i}}-\ddot{\theta}_{i d},
\end{array}\right. \\
& \sum_{\psi i}:=\left\{\begin{array}{l}
\sigma_{1} \dot{\tilde{\psi}}_{1 i}=\widetilde{\psi}_{2 i} \\
\sigma_{1} \dot{\tilde{\psi}}_{2 i}=\frac{K_{\psi i} u_{4 i}}{I_{z i}}-\frac{k_{\psi i} \psi_{2 i}}{I_{z i}}-\ddot{\delta}_{i \psi},
\end{array}\right.
\end{aligned}
$$




$$
\begin{aligned}
& \sum_{x i}:=\left\{\begin{array}{l}
\dot{\tilde{x}}_{1 i}=\widetilde{x}_{2 i} \\
\dot{\tilde{x}}_{2 i}=\frac{\left(\sin \psi_{i} \sin \phi_{i}+\cos \psi_{i} \sin \theta_{i} \cos \phi_{i}\right) u_{1 i}}{m_{i}}-\frac{k_{x i} x_{2 i}}{m_{i}}-\ddot{\delta}_{i x},
\end{array}\right. \\
& \sum_{y i}:=\left\{\begin{array}{l}
\dot{\tilde{y}}_{1 i}=\widetilde{y}_{2 i} \\
\dot{\tilde{y}}_{2 i}=\frac{\left(-\cos \psi_{i} \sin \phi_{i}+\sin \psi_{i} \sin \theta_{i} \cos \phi_{i}\right) u_{1 i}}{m_{i}}-\frac{k_{y i} y_{2 i}}{m_{i}}-\ddot{\delta}_{i y},
\end{array}\right. \\
& \sum_{z i}:=\left\{\begin{array}{l}
\dot{\tilde{z}}_{1 i}=\widetilde{z}_{2 i} \\
\dot{\tilde{z}}_{2 i}=-g+\frac{\left(\cos \theta_{i} \cos \phi_{i}\right) u_{1 i}}{m_{i}}-\frac{k_{z i} z_{2 i}}{m_{i}}-\ddot{\delta}_{i z} .
\end{array}\right.
\end{aligned}
$$

System (9)-(11) has the standard form of a singularly perturbed system with a two-time scale; that is, $a_{i}=$ $\left[e_{1 \phi}^{i}, e_{2 \phi}^{i}, e_{1 \theta}^{i}, e_{2 \theta}^{i}, \widetilde{\psi}_{1 i}, \widetilde{\psi}_{2 i}\right]^{T}$ represents the attitude states with fast time scale and $p_{i}=\left[\widetilde{x}_{1 i}, \tilde{x}_{2 i}, \tilde{y}_{1 i}, \tilde{y}_{2 i}, \widetilde{z}_{1 i}, \widetilde{z}_{2 i}\right]^{T}$ represents the position states with slow time scale. Roughly speaking, in order to maintain a predefined formation shape for multiple quadrotor UAVs, the attitude stabilization should be guaranteed firstly.

3.2. Controller Design. Dynamic event-triggered control scheme is introduced to the multiple quadrotor UAVs. Define the following measurement errors:

$$
\begin{aligned}
& e_{1 \psi}^{i}(t)=\widetilde{\psi}_{1 i}\left(t_{k}^{i}\right)-\widetilde{\psi}_{1 i}(t), \\
& e_{2 \psi}^{i}(t)=\widetilde{\psi}_{2 i}\left(t_{k}^{i}\right)-\widetilde{\psi}_{2 i}(t), \\
& e_{1 x}^{i}(t)=\widetilde{x}_{1 i}\left(t_{k}^{i}\right)-\widetilde{x}_{1 i}(t), \\
& e_{2 x}^{i}(t)=\widetilde{x}_{2 i}\left(t_{k}^{i}\right)-\widetilde{x}_{2 i}(t), \\
& e_{1 y}^{i}(t)=\tilde{y}_{1 i}\left(t_{k}^{i}\right)-\widetilde{y}_{1 i}(t), \\
& e_{2 y}^{i}(t)=\widetilde{y}_{2 i}\left(t_{k}^{i}\right)-\widetilde{y}_{2 i}(t), \\
& e_{1 z}^{i}(t)=\widetilde{z}_{1 i}\left(t_{k}^{i}\right)-\widetilde{z}_{1 i}(t), \\
& e_{2 z}^{i}(t)=\widetilde{z}_{2 i}\left(t_{k}^{i}\right)-\widetilde{z}_{2 i}(t) .
\end{aligned}
$$

Let

$$
\begin{aligned}
& \psi_{p i}=\sum_{j \in \Omega_{i}^{\omega}} a_{i j}^{\omega}\left(\widetilde{\psi}_{1 i}\left(t_{k}^{i}\right)-\widetilde{\psi}_{1 j}\left(t_{k^{\prime}}^{j}\right)\right)+a_{i i}^{l} \widetilde{\psi}_{1 i}\left(t_{k}^{i}\right), \\
& \psi_{v i}=\sum_{j \in \Omega_{i}^{\omega}} a_{i j}^{\omega}\left(\widetilde{\psi}_{2 i}\left(t_{k}^{i}\right)-\widetilde{\psi}_{2 j}\left(t_{k^{\prime}}^{j}\right)\right)+a_{i i}^{l} \widetilde{\psi}_{2 i}\left(t_{k}^{i}\right), \\
& x_{p i}=\sum_{j \in \Omega_{i}^{\omega}} a_{i j}^{\omega}\left(\widetilde{x}_{1 i}\left(t_{k}^{i}\right)-\widetilde{x}_{1 j}\left(t_{k^{\prime}}^{j}\right)\right)+a_{i i}^{l} \widetilde{x}_{1 i}\left(t_{k}^{i}\right), \\
& x_{v i}=\sum_{j \in \Omega_{i}^{\omega}} a_{i j}^{\omega}\left(\widetilde{x}_{2 i}\left(t_{k}^{i}\right)-\widetilde{x}_{2 j}\left(t_{k^{\prime}}^{j}\right)\right)+a_{i i}^{l} \widetilde{x}_{2 i}\left(t_{k}^{i}\right),
\end{aligned}
$$

$$
\begin{aligned}
& y_{p i}=\sum_{j \in \Omega_{i}^{\omega}} a_{i j}^{\omega}\left(\tilde{y}_{1 i}\left(t_{k}^{i}\right)-\tilde{y}_{1 j}\left(t_{k^{\prime}}^{j}\right)\right)+a_{i i}^{l} \tilde{y}_{1 i}\left(t_{k}^{i}\right), \\
& y_{v i}=\sum_{j \in \Omega_{i}^{\omega}} a_{i j}^{\omega}\left(\tilde{y}_{2 i}\left(t_{k}^{i}\right)-\tilde{y}_{2 j}\left(t_{k^{\prime}}^{j}\right)\right)+a_{i i}^{l} \tilde{y}_{2 i}\left(t_{k}^{i}\right), \\
& z_{p i}=\sum_{j \in \Omega_{i}^{\omega}} a_{i j}^{\omega}\left(\widetilde{z}_{1 i}\left(t_{k}^{i}\right)-\widetilde{z}_{1 j}\left(t_{k^{\prime}}^{j}\right)\right)+a_{i i}^{l} \widetilde{z}_{1 i}\left(t_{k}^{i}\right), \\
& z_{v i}=\sum_{j \in \Omega_{i}^{\omega}} a_{i j}^{\omega}\left(\widetilde{z}_{2 i}\left(t_{k}^{i}\right)-\widetilde{z}_{2 j}\left(t_{k^{\prime}}^{j}\right)\right)+a_{i i}^{l} \widetilde{z}_{2 i}\left(t_{k}^{i}\right) .
\end{aligned}
$$

Then, the auxiliary control variables are given by

$$
\begin{aligned}
& v_{\phi i}=-\alpha_{\phi} e_{1 \phi}^{i}-\beta_{\phi} e_{2 \phi}^{i}, \\
& v_{\theta i}=-\alpha_{\theta} e_{1 \theta}^{i}-\beta_{\theta} e_{2 \theta}^{i}, \\
& v_{\psi i}=-\alpha_{\psi} \psi_{p i}-\beta_{\psi} \psi_{v i}, \\
& v_{x i}=-\alpha_{x} x_{p i}-\beta_{x} x_{v i}, \\
& v_{y i}=-\alpha_{y} y_{p i}-\beta_{y} y_{v i}, \\
& v_{z i}=-\alpha_{z} z_{p i}-\beta_{z} z_{v i} .
\end{aligned}
$$

Therefore, the choice of control inputs

$$
\begin{aligned}
& u_{2 i}=\frac{I_{x i} v_{\phi i}}{l_{i}}+k_{\phi i} \phi_{2 i}+\frac{I_{x i} \ddot{\phi}_{i d}}{l_{i}}, \\
& u_{3 i}=\frac{I_{y i} v_{\theta i}}{l_{i}}+k_{\theta i} \theta_{2 i}+\frac{I_{y i} \ddot{\theta}_{i d}}{l_{i}}, \\
& u_{4 i}=\frac{I_{z i} v_{\psi i}}{K_{\psi i}}+\frac{k_{\psi i} \psi_{2 i}}{K_{\psi i}}+\frac{I_{z i} \ddot{\delta}_{i \psi}}{K_{\psi i}}, \\
& u_{1 i}=\frac{m_{i} v_{z i}+m \ddot{\delta}_{i z}+m_{i} g+k_{z i} z_{2 i}}{\cos \theta_{i d} \cos \phi_{i d}},
\end{aligned}
$$

where the reference angles $\phi_{i d}$ and $\theta_{i d}$ are addressed as 


$$
\begin{aligned}
& \theta_{i d}=\tan ^{-1} \frac{\cos \psi_{i d}\left(v_{x i}+\ddot{\delta}_{i x}+k_{x i} x_{2 i} / m_{i}\right)+\sin \psi_{i d}\left(v_{y i}+\ddot{\delta}_{i y}+k_{y i} y_{2 i} / m_{i}\right)}{v_{z i}+g+\ddot{\delta}_{i z}+k_{z i} z_{2 i} / m_{i}}, \\
& \phi_{i d}=\tan ^{-1} \frac{\cos \theta_{i d}\left(\sin \psi_{i d}\left(v_{x i}+\ddot{\delta}_{i x}+k_{x i} x_{2 i} / m_{i}\right)-\cos \psi_{i d}\left(v_{y i}+\ddot{\delta}_{i y}+k_{y i} y_{2 i} / m_{i}\right)\right)}{v_{z i}+g+\ddot{\delta}_{i z}+k_{z i} z_{2 i} / m_{i}} .
\end{aligned}
$$

Remark 7. It should be stressed that (17) and (18) are needed to be nonsingular; in other words, the denominator of (17) or (18) cannot be zero. In practice, the quadrotor has to take a certain thrust to overcome gravity in order to maintain hovering; otherwise, it would sink vertically. It means that the denominator of (17) or (18) is approximate to $g$, and during the flight, it will also be greater than zero based on the defined domain of $z$ dynamics $\left[z_{1 i}, z_{2 i}, \dot{z}_{2 i}\right]^{T} \in D_{z}=\mathbb{R} \times\left\{\left|z_{2 i}\right|<\right.$ $\left.d_{z 2}\right\} \times\left\{\left|\dot{z}_{2 i}\right|<d_{\dot{z} 2}\right\} \subset \mathbb{R}^{3}, d_{\dot{z} 2} \ll g$.

3.3. Dynamic Event-Triggered Communication. Figure 2 depicts the control law running in the ith quadrotor. Generally speaking, each agent updates its controller whenever the designed trigger condition is reached, called the triggered event. Based on local information, it decides when to broadcast its current state over the network. In other words, the key problem is to find a triggering rule that determines when agent $i$ has to broadcast the new state information to its neighbors.

In contrast to most of the existing works, a new dynamic event-triggered communication mechanism is developed to schedule interagent communication. The threshold parameter in the proposed event triggering condition will not be fixed permanently but vary with time by following a dynamic rule. The detailed dynamic rule is provided in the following section. The event-triggered control strategy works as follows: define a trigger function $f_{i}(t)$ for each agent, which depends on local information only; an event is triggered as soon as the trigger condition $f_{i}(t)>0$ is fulfilled, while each agent recomputes its control law in accordance with the measurement error, such that all the agents could reach and keep the predesigned formation shape. It will be shown numerically that the dynamic event-triggered communication mechanism can achieve a better tradeoff between reducing data transmissions and preserving favorable formation performance.

3.4. System Stability via Two-Time Scale. The stability analysis for each subsystem will be done by starting from the faster one to the slower one. The main results of this paper are presented next with the help of the following theorems.

Theorem 8. Consider the ith quadrotor dynamics, which is also a singularly perturbed system. Then, there always exists $\sigma_{1}^{*}>0$ such that the state space origin of the closed-loop system (9)-(11) is exponentially stable with $\sigma_{1}^{*}>\sigma_{1}>0$.

Proof. The proof is set down in the following five items.

(1) System (9)-(11) has a unique equilibrium point at $\left[a_{i}^{T}, p_{i}^{T}\right]^{T}=0$.

(2) The quasi-steady-state solution of closed-loop system (9)-(10) is $a_{i}=a^{*}=h\left(t, p_{i}\right)$. Hence, by substituting $\sigma_{1}=$ 0 into (9)-(10), the isolated root is given by $a^{*}=0$. It is noteworthy that the isolated root evaluated in (16)-(18) gives the specific values $\phi_{i d}^{*}, \theta_{i d}^{*}$, and $\psi_{i d}^{*}$.

(3) System (9)-(11) and the isolated root have bounded partial derivatives in compact sets.

(4) By considering $\sigma_{1}=0$ and using the isolated root into (11), the following slow dynamics system is obtained:

$$
\begin{aligned}
& \sum_{x i}:=\left\{\begin{array}{l}
\dot{\tilde{x}}_{1 i}=\tilde{x}_{2 i} \\
\dot{\tilde{x}}_{2 i}=\frac{\left(\sin \psi_{i d}^{*} \sin \phi_{i d}^{*}+\cos \psi_{i d}^{*} \sin \theta_{i d}^{*} \cos \phi_{i d}^{*}\right) u_{1 i}}{m_{i}}-\frac{k_{x i} x_{2 i}}{m_{i}}-\ddot{\delta}_{i x},
\end{array}\right. \\
& \sum_{y i}:=\left\{\begin{array}{l}
\dot{\tilde{y}}_{1 i}=\tilde{y}_{2 i} \\
\dot{\tilde{y}}_{2 i}=\frac{\left(-\cos \psi_{i d}^{*} \sin \phi_{i d}^{*}+\sin \psi_{i d}^{*} \sin \theta_{i d}^{*} \cos \phi_{i d}^{*}\right) u_{1 i}}{m_{i}}-\frac{k_{y i} y_{2 i}}{m_{i}}-\ddot{\delta}_{i y},
\end{array}\right. \\
& \sum_{z i}:=\left\{\begin{array}{l}
\dot{\tilde{z}}_{1 i}=\widetilde{z}_{2 i} \\
\dot{\tilde{z}}_{2 i}=-g+\frac{\left(\cos \theta_{i d}^{*} \cos \phi_{i d}^{*}\right) u_{1 i}}{m_{i}}-\frac{k_{z i} z_{2 i}}{m_{i}}-\ddot{\delta}_{i z} .
\end{array}\right.
\end{aligned}
$$

The local stability of the slow dynamics (11) can be derived by using event-triggered control method. For the sake of simplicity, it will be given in Theorem 12 later.
(5) To obtain the boundary layer system, by deriving $a-a^{*}$ with respect to scaled time $\kappa=t / \sigma_{1}$ and setting $\sigma_{1}=0$, the boundary layer system is obtained: 


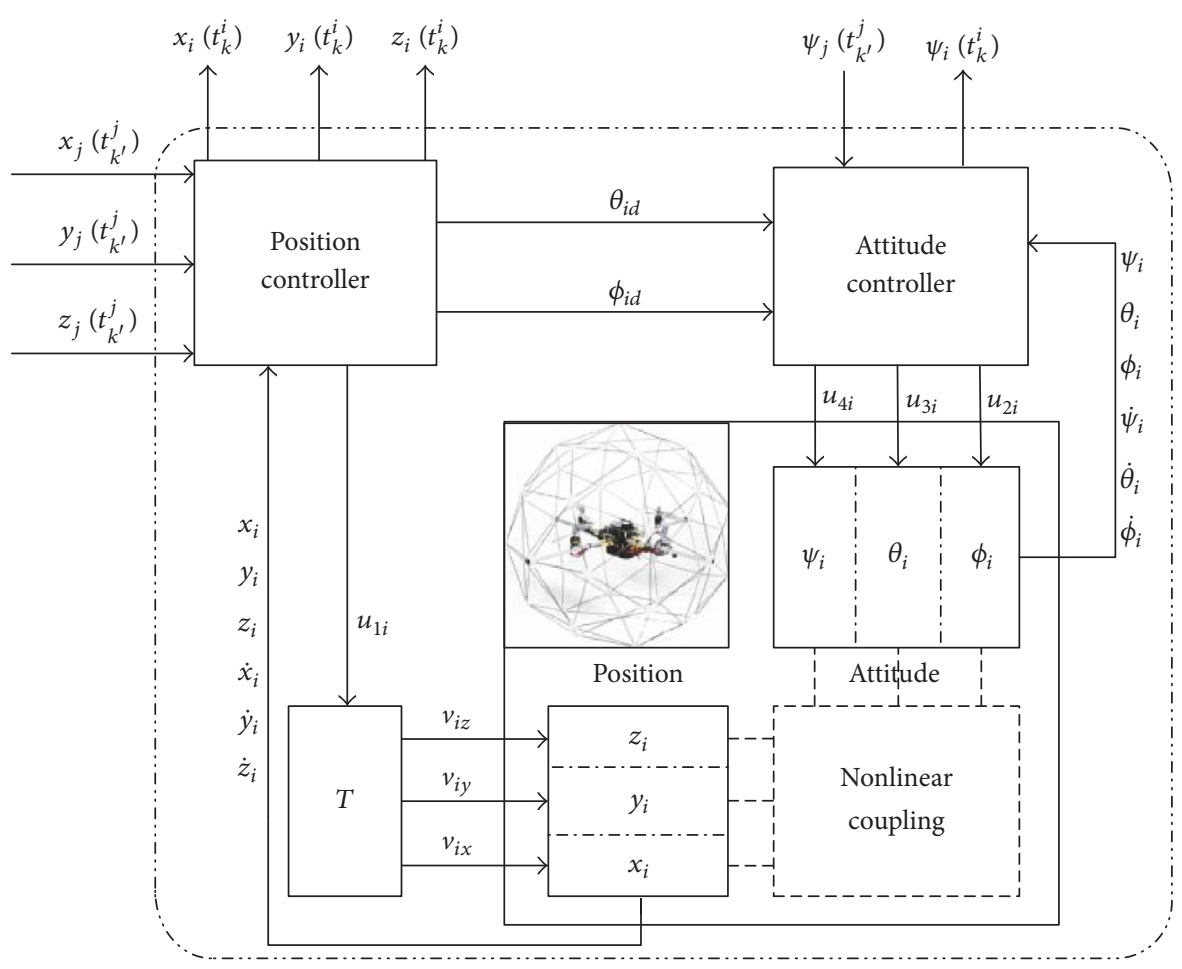

FIGURE 2: Block diagram of the event-based control strategy.

$$
\begin{aligned}
& \frac{d}{d \kappa} e_{1 \phi}^{i}=e_{2 \phi}^{i}, \\
& \frac{d}{d \kappa} e_{2 \phi}^{i}=-\alpha_{\phi} e_{1 \phi}^{i}-\beta_{\phi} e_{2 \phi}^{i}, \\
& \frac{d}{d \kappa} e_{1 \theta}^{i}=e_{2 \theta}^{i}, \\
& \frac{d}{d \kappa} e_{2 \theta}^{i}=-\alpha_{\theta} e_{1 \theta}^{i}-\beta_{\theta} e_{2 \theta}^{i}, \\
& \frac{d}{d \kappa} \widetilde{\psi}_{1 i}=\widetilde{\psi}_{2 i}, \\
& \frac{d}{d \kappa} \widetilde{\psi}_{2 i}=-\alpha_{\psi} \psi_{p i}-\beta_{\psi} \psi_{v i} .
\end{aligned}
$$

Thus, for the $\phi_{i}$ and $\theta_{i}$ subsystems

$$
\begin{aligned}
\frac{d}{d \kappa} e_{\phi}^{i} & =A_{\phi} e_{\phi}^{i} \Longrightarrow \\
\frac{d}{d \kappa}\left[\begin{array}{c}
e_{1 \phi}^{i} \\
e_{2 \phi}^{i}
\end{array}\right] & =\left[\begin{array}{cc}
0 & 1 \\
-\alpha_{\phi} & -\beta_{\phi}
\end{array}\right]\left[\begin{array}{c}
e_{1 \phi}^{i} \\
e_{2 \phi}^{i}
\end{array}\right], \\
\frac{d}{d \kappa} e_{\theta}^{i} & =A_{\theta} e_{\theta}^{i} \Longrightarrow \\
\frac{d}{d \kappa}\left[\begin{array}{c}
e_{1 \theta}^{i} \\
e_{2 \theta}^{i}
\end{array}\right] & =\left[\begin{array}{cc}
0 & 1 \\
-\alpha_{\theta} & -\beta_{\theta}
\end{array}\right]\left[\begin{array}{c}
e_{1 \theta}^{i} \\
e_{2 \theta}^{i}
\end{array}\right] .
\end{aligned}
$$

It is obvious that $A_{\phi}$ and $A_{\theta}$ are Hurwitz, where system (22) is exponentially stable. Meanwhile, the stability result of $\psi_{i}$ subsystem will be established in Theorem 10 as follows.

According to [32], there are sufficient conditions to claim that there exists $\sigma_{1}^{*}$ such that $\sigma_{1}^{*}>\sigma_{1}>0$ guarantees that system (9)-(11) achieves the following $\operatorname{limit}_{\lim _{t \rightarrow \infty}}\left[a_{i}^{T}, p_{i}^{T}\right]^{T}=$ 0 .

Remark 9. Based on the system stability analysis by the singular perturbation method, instead of using the feedback angles $\phi_{i}, \theta_{i}, \psi_{i}$, the control input $u_{1 i}$ is related to the reference angles $\phi_{i d}, \theta_{i d}, \psi_{i d}$, and the coupling between the attitude subsystem and the position subsystem is diminished.

Theorem 10. Given $\widetilde{\psi}_{p i}=\sum_{j \in \Omega_{i}^{\omega}} a_{i j}^{\omega}\left(e_{1 \psi}^{i}-e_{1 \psi}^{j}\right)+a_{i i}^{l} e_{1 \psi}^{i}$ and $\widetilde{\psi}_{v i}=\sum_{j \in \Omega_{i}^{\omega}} a_{i j}^{\omega}\left(e_{2 \psi}^{i}-e_{2 \psi}^{j}\right)+a_{i i}^{l} e_{2 \psi}^{i}$, then define $E_{\psi}^{i}=\left[\widetilde{\psi}_{p i}\right.$, $\left.\widetilde{\psi}_{v i}\right]^{T}$ and $\widetilde{\Psi}_{i}=\left[\psi_{p i}-\widetilde{\psi}_{p i}, \psi_{v i}-\widetilde{\psi}_{v i}\right]^{T}$. Consider the yaw subsystem (21); the system stability could be obtained through the distributed control law (14) and (15) and the following eventtriggered communication condition:

$$
f_{i}(t)=\left\|B_{2}^{T} Q E_{\psi}^{i}\right\|-\sigma(t)\left\|B_{2}^{T} Q \widetilde{\Psi}_{i}\right\|
$$

where $\sigma(t)=e^{-\mu e_{s}}$ is the dynamic threshold parameter with $e_{s}=\int_{0}^{t_{k}^{i}} e_{\psi}^{i} e_{\psi}^{T} d s, e_{\psi}^{i}=\left[e_{1 \psi}^{i}, e_{2 \psi}^{i}\right]^{T}, \mu>0$, and the initial value $\sigma(0)=\sigma_{0} \in(0,1)$, which ensures that $f_{i}(t) \leq 0$ always holds.

Proof. Obviously, $\sigma(t)$ is a monotone nonincreasing function; thus, $\sigma(t) \leq \sigma_{0}$ always holds. Particularly, if the threshold 
parameter is preset as a constant $\sigma_{0}$ before dynamic eventtriggered controller is implemented, this is called the static event-triggered controller. Define $B_{1}=[1,0]^{T}, B_{2}=[0,1]^{T}$, and $\widetilde{\psi}_{i}=\left[\widetilde{\psi}_{1 i}, \widetilde{\psi}_{2 i}\right]^{T}$. Based on (13), (21) becomes

$$
\frac{d}{d \kappa} \widetilde{\psi}_{i}=B_{1} B_{2}^{T} \widetilde{\psi}_{i}+B_{2}\left(-\alpha_{\psi} \psi_{p i}-\beta_{\psi} \psi_{v i}\right)
$$

Hence, it follows from [35] that there exist positive constants $\alpha_{0}, \alpha_{\psi}$, and $\beta_{\psi}$ such that

$$
\begin{aligned}
\frac{1}{2}\left(Q B_{1} B_{2}^{T}+B_{2} B_{1}^{T} Q\right)-\rho_{0} Q B_{2}^{T} B_{2} Q+2 I_{2} \leq 0 & \\
& Q>0,
\end{aligned}
$$

where $Q=\left[\alpha_{0}, \alpha_{\psi} ; \alpha_{\psi}, \beta_{\psi}\right], B_{2}^{T} Q=\left[\alpha_{\psi}, \beta_{\psi}\right]$, and

$$
0<\rho_{0} \leq\left(1-\frac{\rho_{1}}{2}-\frac{\sigma_{0}^{2}}{2 \rho_{1}}\right) \lambda_{\text {min }}\left(L^{G}\right) \text {. }
$$

Let $\Psi=\left[\widetilde{\psi}_{1}, \ldots, \widetilde{\psi}_{N}\right]^{T}$ and $E_{\psi}=\left[e_{\psi}^{1}, \ldots, e_{\psi}^{N}\right]^{T}$. Consider the following Lyapunov function:

$$
V_{\psi}=\frac{1}{2} \Psi^{T}\left(I_{N} \otimes Q\right) \Psi
$$

Then, its derivative is

$$
\begin{aligned}
\frac{d}{d \kappa} V_{\psi}= & \Psi^{T}\left(I_{N} \otimes \frac{Q B_{1} B_{2}^{T}+B_{2} B_{1}^{T} Q}{2}\right) \Psi \\
& -\Psi^{T}\left(L^{G} \otimes Q B_{2} B_{2}^{T} Q\right) \Psi \\
& -\Psi^{T}\left(L^{G} \otimes Q B_{2} B_{2}^{T} Q\right) E_{\psi} .
\end{aligned}
$$

Based on Lemma 6, the following inequality holds:

$$
\begin{gathered}
\Psi^{T}\left(L^{G} \otimes Q B_{2} B_{2}^{T} Q\right) E_{\psi} \\
\leq \frac{\rho_{1}}{2} \Psi^{T}\left(L^{G} \otimes Q B_{2} B_{2}^{T} Q\right) \Psi \\
\quad+\frac{1}{2 \rho_{1}} E_{\psi}^{T}\left(L^{G} \otimes Q B_{2} B_{2}^{T} Q\right) E_{\psi} .
\end{gathered}
$$

Based on (23) and (25), (28) can be written as

$$
\begin{aligned}
& \frac{d}{d \kappa} V_{\psi} \leq \Psi^{T}\left(I_{N} \otimes \frac{Q B_{1} B_{2}^{T}+B_{1} B_{2}^{T} Q^{T}}{2}\right) \Psi \\
& \quad-\lambda_{\min }\left(L^{G}\right) \Psi^{T}\left(I_{N} \otimes Q B_{2} B_{2}^{T} Q\right) \Psi+\frac{\rho_{1}}{2} \Psi^{T}\left(L^{G}\right. \\
& \left.\otimes Q B_{2} B_{2}^{T} Q\right) \Psi+\frac{1}{2 \rho_{1}} E_{\psi}^{T}\left(L^{G} \otimes Q B_{2} B_{2}^{T} Q\right) E_{\psi} \\
& \quad \leq \Psi^{T}\left(I _ { N } \otimes \left[\frac{Q B_{1} B_{2}^{T}+B_{1} B_{2}^{T} Q^{T}}{2}\right.\right. \\
& \left.\left.\quad-\left(1-\frac{\rho_{1}}{2}-\frac{\sigma^{2}(t)}{2 \rho_{1}}\right) \lambda_{\min }\left(L^{G}\right) Q B_{2} B_{2}^{T} Q\right]\right) \Psi \\
& \leq-2 \rho(Q) V_{\psi} .
\end{aligned}
$$

Hence, $V_{\psi} \leq \sigma_{1} V_{\psi}(0) e^{-2 \rho(Q) t}$, and $\lim _{t \rightarrow \infty}\left(\psi_{1 i}(t)-\delta_{i \psi}(t)-\right.$ $\left.\psi_{d}(t)\right)=0$ exponentially.

Next, Zeno behavior is excluded. For any $t>t_{k}^{i}$, it follows from (23) that

$$
\begin{aligned}
& \left\|\frac{d}{d \kappa}\left(L^{G} \otimes B_{2}^{T} Q\right) E_{\psi}\right\| \leq \sqrt{\alpha_{\psi}^{2}+\beta_{\psi}^{2}}\left\|L^{G}\right\|\left(\left\|E_{\psi}\right\|\right. \\
& \left.+\sqrt{\alpha_{\psi}^{2}+\beta_{\psi}^{2}}\left\|L^{G}\right\|\|\Psi\|+\sqrt{\alpha_{\psi}^{2}+\beta_{\psi}^{2}}\left\|L^{G}\right\|\left\|E_{\psi}\right\|\right) \\
& \leq\left(\sigma(t)+(1+\sigma(t)) \sqrt{\alpha_{\psi}^{2}+\beta_{\psi}^{2}}\left\|L^{G}\right\|\right) \\
& \cdot \sqrt{\alpha_{\psi}^{2}+\beta_{\psi}^{2}}\left\|L^{G}\right\|\|\Psi\|,
\end{aligned}
$$

where $\|\Psi\| \leq \sqrt{2 \sigma_{1} \rho(Q) V_{\psi}(0)} e^{-\rho(Q) t}$. Hence,

$$
\begin{aligned}
& \left\|\frac{d}{d \kappa}\left(L^{G} \otimes B_{2}^{T} Q\right) E_{\psi}\right\| \\
& \leq\left(\sigma(t)+(1+\sigma(t)) \sqrt{\alpha_{\psi}^{2}+\beta_{\psi}^{2}}\left\|L^{G}\right\|\right) \\
& \cdot \sqrt{\alpha_{\psi}^{2}+\beta_{\psi}^{2}}\left\|L^{G}\right\| \sqrt{2 \sigma_{1} \rho(Q) V_{\psi}(0)} e^{-\rho(Q) t} .
\end{aligned}
$$

Thus,

$$
\begin{aligned}
& \left\|\left(L^{G} \otimes B_{2}^{T} Q\right) E_{\psi}\right\| \\
& \leq \sigma_{1}\left(\sigma(t)+(1+\sigma(t)) \sqrt{\alpha_{\psi}^{2}+\beta_{\psi}^{2}}\left\|L^{G}\right\|\right) \\
& \cdot \sqrt{\alpha_{\psi}^{2}+\beta_{\psi}^{2}}\left\|L^{G}\right\| \sqrt{2 \sigma_{1} \rho(Q) V_{\psi}(0)} e^{-\rho(Q) t}\left(t-t_{k}\right) .
\end{aligned}
$$

The next event $t_{k+1}$ will not be triggered before $\|\left(L^{G} \otimes\right.$ $\left.B_{2}^{T} Q\right) E_{\psi}\|=\sigma(t)\|\left(L^{G} \otimes B_{2}^{T} Q\right) \Psi \|$. Thus, a lower bound on the interevent intervals is given by $\tau=t-t_{k}$ that solves the following equation:

$$
\begin{gathered}
\sigma(\tau)\left\|\left(L^{G} \otimes B_{2}^{T} Q\right) \Psi\right\| \\
=\sigma_{1} \tau\left(\sigma(\tau)+(1+\sigma(\tau)) \sqrt{\alpha_{\psi}^{2}+\beta_{\psi}^{2}}\left\|L^{G}\right\|\right) \\
\cdot \sqrt{\alpha_{\psi}^{2}+\beta_{\psi}^{2}}\left\|L^{G}\right\| \sqrt{2 \sigma_{1} \rho(Q) V_{\psi}(0)} e^{-\rho(Q) t} .
\end{gathered}
$$

Therefore, for all $t_{k} \geq 0$ the solutions $\tau\left(t_{k}\right)$ are greater than or equal to $\tau$ given by

$$
\sigma(\tau)=\sigma_{1} \tau\left(\sigma(\tau)+(1+\sigma(\tau)) \sqrt{\alpha_{\psi}^{2}+\beta_{\psi}^{2}}\left\|L^{G}\right\|\right)
$$

which is strictly positive, so Zeno behavior is excluded. This completes the proof.

Remark 11. It should be pointed out that the vectors $\phi_{i d}$ and $\theta_{i d}$ are obtained inside of the controller; as shown in Figure 2, the pitch and roll attitude dynamics are not eventtriggered. In other words, only the yaw angle in the attitude subsystem is controlled through an event-triggered strategy. In addition, compared with some existing works, in which 
assuming $\psi_{1 i}=0$ in the position control loop, the yaw angle is not always zero in practice. Since that the control method is more suitable in this paper.

After the attitude angles reach quasi-steady states, hierarchically, $\sigma_{1}=0, \phi_{1 i} \rightarrow \phi_{i d}, \theta_{1 i} \rightarrow \theta_{i d}, \psi_{1 i} \rightarrow \psi_{i d}$. Substituting (14), (16)-(18) into (19), it is possible to show that the closedloop position subsystem can be written as

$$
\begin{aligned}
& \dot{\tilde{x}}_{1 i}=\tilde{x}_{2 i}, \\
& \dot{\tilde{x}}_{2 i}=-\alpha_{x} x_{p i}-\beta_{x} x_{v i}, \\
& \dot{\tilde{y}}_{1 i}=\tilde{y}_{2 i}, \\
& \dot{\tilde{y}}_{2 i}=-\alpha_{y} y_{p i}-\beta_{y} y_{v i}, \\
& \dot{\tilde{z}}_{1 i}=\widetilde{z}_{2 i}, \\
& \dot{\tilde{z}}_{2 i}=-\alpha_{z} z_{p i}-\beta_{z} z_{v i} .
\end{aligned}
$$

Without loss of generality, we only provide the proof along $x$-subsystem in the following.

Theorem 12. Given $\tilde{x}_{p i}=\sum_{j \in \Omega_{i}^{\omega}} a_{i j}^{\omega}\left(e_{1 x}^{i}-e_{1 x}^{j}\right)+a_{i i}^{l} e_{1 x}^{i}$ and $\tilde{x}_{v i}=\sum_{j \in \Omega_{i}^{\omega}} a_{i j}^{\omega}\left(e_{2 x}^{i}-e_{2 x}^{j}\right)+a_{i i}^{l} e_{2 x}^{i}$, then let $E_{x}^{i}=\left[\tilde{x}_{p i}, \tilde{x}_{v i}\right]^{T}$ and $\widetilde{X}_{i}=\left[x_{p i}-\tilde{x}_{p i}, x_{v i}-\tilde{x}_{v i}\right]^{T}$. Considering the position subsystem (19), the system stability could be obtained through the distributed control law (16)-(18) and the following eventtriggered communication condition:

$$
f_{i}(t)=\left\|B_{2}^{T} Q_{0} E_{x}^{i}\right\|-\sigma(t)\left\|B_{2}^{T} Q_{0} \widetilde{X}_{i}\right\|
$$

where $\sigma(t)=e^{-\mu e_{s}}$ is the dynamic threshold parameter with $e_{s}=\int_{0}^{t_{k}^{i}} e_{x}^{i^{T}} e_{x}^{i} d s, e_{x}^{i}=\left[e_{1 x}^{i}, e_{2 x}^{i}\right]^{T}, \mu>0$ and the initial value $\sigma(0)=\sigma_{0} \in(0,1)$.

Proof. Let $\tilde{x}_{i}=\left[\tilde{x}_{1 i}, \tilde{x}_{2 i}\right]^{T}$; the $x$-subsystem could be rewritten as

$$
\dot{\tilde{x}}_{i}=B_{1} B_{2}^{T} \tilde{x}_{i}+B_{2}\left(-\alpha_{x} x_{p i}-\beta_{x} x_{v i}\right) .
$$

Similarly, there exist positive constants $\alpha_{0}, \alpha_{x}$, and $\beta_{x}$ such that

$$
\begin{aligned}
\frac{1}{2}\left(Q_{0} B_{1} B_{2}^{T}+B_{2} B_{1}^{T} Q_{0}\right)-\rho_{0} Q_{0} B_{2}^{T} B_{2} Q_{0}+2 I_{2} & \leq 0 \\
& Q_{0}>0
\end{aligned}
$$

where $Q_{0}=\left[\alpha_{0}, \alpha_{x} ; \alpha_{x}, \beta_{x}\right]$. Note that $B_{2}^{T} Q_{0}=\left[\alpha_{x}, \beta_{x}\right]$, and let $X=\left[\widetilde{x}_{1}, \ldots, \widetilde{x}_{N}\right]^{T}$ and $E_{X}=\left[e_{x}^{1}, \ldots, e_{x}^{N}\right]^{T}$. Design the following Lyapunov function:

$$
V_{x}=\frac{1}{2} X^{T}\left(I_{N} \otimes Q_{0}\right) X
$$

Then, its derivative is

$$
\begin{aligned}
\dot{V}_{x}= & X^{T}\left(I_{N} \otimes \frac{Q_{0} B_{1} B_{2}^{T}+B_{2} B_{1}^{T} Q_{0}}{2}\right) X \\
& -X^{T}\left(L^{G} \otimes Q_{0} B_{2} B_{2}^{T} Q_{0}\right) X \\
& -X^{T}\left(L^{G} \otimes Q_{0} B_{2} B_{2}^{T} Q_{0}\right) E_{X} .
\end{aligned}
$$

Based on Lemma 6, the following inequality holds:

$$
\begin{aligned}
X^{T}\left(L^{G} \otimes Q_{0} B_{2} B_{2}^{T} Q_{0}\right) E_{X} \\
\leq \frac{\rho_{1}}{2} X^{T}\left(L^{G} \otimes Q_{0} B_{2} B_{2}^{T} Q_{0}\right) X \\
\quad+\frac{1}{2 \rho_{1}} E_{X}^{T}\left(L^{G} \otimes Q_{0} B_{2} B_{2}^{T} Q_{0}\right) E_{X}
\end{aligned}
$$

Based on (37) and (39), (41) can be written as

$$
\begin{aligned}
\dot{V}_{x} & \leq X^{T}\left(I _ { N } \otimes \left[\frac{Q_{0} B_{1} B_{2}^{T}+B_{1} B_{2}^{T} Q_{0}^{T}}{2}\right.\right. \\
& \left.\left.-\left(1-\frac{\rho_{1}}{2}-\frac{\sigma^{2}(t)}{2 \rho_{1}}\right) \lambda_{\min }\left(L^{G}\right) Q_{0} B_{2} B_{2}^{T} Q_{0}\right]\right) X \\
& \leq-2 \rho\left(Q_{0}\right) V_{x} .
\end{aligned}
$$

Hence, $V_{x} \leq V_{x}(0) e^{-2 \rho\left(\mathrm{Q}_{0}\right) t}$, and $\lim _{t \rightarrow \infty}\left(x_{1 i}(t)-\delta_{i x}(t)-\right.$ $\left.x_{d}(t)\right)=0$ exponentially.

Next, Zeno behavior is excluded. For any $t>t_{k}^{i}$, one has

$$
\begin{aligned}
& \left\|\frac{d}{d t}\left(L^{G} \otimes B_{2}^{T} Q\right) E_{X}\right\| \leq \sqrt{\alpha_{x}^{2}+\beta_{x}^{2}}\left\|L^{G}\right\|\left(\left\|E_{X}\right\|\right. \\
& \left.+\sqrt{\alpha_{x}^{2}+\beta_{x}^{2}}\left\|L^{G}\right\|\|X\|+\sqrt{\alpha_{x}^{2}+\beta_{x}^{2}}\left\|L^{G}\right\|\left\|E_{X}\right\|\right) \\
& \leq\left(\sigma(t)+(1+\sigma(t)) \sqrt{\alpha_{x}^{2}+\beta_{x}^{2}}\left\|L^{G}\right\|\right) \\
& \cdot \sqrt{\alpha_{x}^{2}+\beta_{x}^{2}}\left\|L^{G}\right\|\|X\|,
\end{aligned}
$$

where $\|X\| \leq \sqrt{2 \rho\left(Q_{0}\right) V_{x}(0)} e^{-\rho\left(Q_{0}\right) t}$. Hence,

$$
\begin{aligned}
& \left\|\frac{d}{d t}\left(L^{G} \otimes B_{2}^{T} Q\right) E_{X}\right\| \\
& \leq\left(\sigma(t)+(1+\sigma(t)) \sqrt{\alpha_{x}^{2}+\beta_{x}^{2}}\left\|L^{G}\right\|\right) \\
& \cdot \sqrt{\alpha_{x}^{2}+\beta_{x}^{2}}\left\|L^{G}\right\| \sqrt{2 \rho\left(Q_{0}\right) V_{x}(0)} e^{-\rho\left(Q_{0}\right) t} .
\end{aligned}
$$

Thus,

$$
\begin{aligned}
& \left\|\left(L^{G} \otimes B_{2}^{T} Q\right) E_{X}\right\| \\
& \quad \leq\left(\sigma(t)+(1+\sigma(t)) \sqrt{\alpha_{x}^{2}+\beta_{x}^{2}}\left\|L^{G}\right\|\right) \\
& \cdot \sqrt{\alpha_{x}^{2}+\beta_{x}^{2}}\left\|L^{G}\right\| \sqrt{2 \rho\left(Q_{0}\right) V_{x}(0)} e^{-\rho\left(Q_{0}\right) t}\left(t-t_{k}\right) .
\end{aligned}
$$




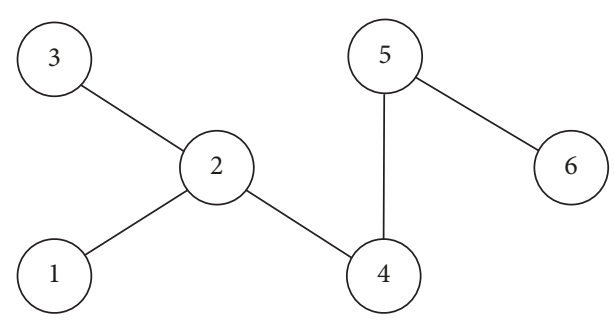

FIGURE 3: Initial mode of interaction topology.

The next event $t_{k+1}$ will not be triggered before $\|\left(L^{G} \otimes\right.$ $\left.B_{2}^{T} Q\right) E_{X}\|=\sigma(t)\|\left(L^{G} \otimes B_{2}^{T} Q\right) X \| ;$ it means

$$
\begin{aligned}
& \sigma(t)\left\|\left(L^{G} \otimes B_{2}^{T} Q\right) X\right\| \\
& \leq\left(\sigma(t)+(1+\sigma(t)) \sqrt{\alpha_{x}^{2}+\beta_{x}^{2}}\left\|L^{G}\right\|\right) \\
& \cdot \sqrt{\alpha_{x}^{2}+\beta_{x}^{2}}\left\|L^{G}\right\| \\
& \cdot \sqrt{2 \rho\left(Q_{0}\right) V_{x}(0)} e^{-\rho\left(Q_{0}\right) t}\left(t_{k+1}-t_{k}\right) .
\end{aligned}
$$

By the same graphical argument as in the proof of Theorem 10, it can be concluded that a lower bounded on the interevent times is given by $\tau$, so Zeno behavior is excluded.

This completes the proof.

Remark 13. Compared with the existing works, the threshold parameter of event triggering condition will not be fixed permanently but vary with time by following the measurement errors. Since that it can achieve a better tradeoff between reducing event times and preserving favorable formation performance.

\section{Simulation Results}

In this section, simulations are given to demonstrate the effectiveness of the theoretical results. Consider a group of agents, which consists of six quadrotor UAVs, and the dynamics can be written as (4). The system parameters are shown in Table 1. Furthermore, the initial mode of undirected topology $G(\omega(t))$ is given in Figure 3. A special case is considered, where $\omega_{0}$ takes the switch value in $S_{0}=\{1,2,3,4$, $5,6\}$ with an equal probability $1 / 6$ and the time interval $\Delta_{0}=$ $5 \mathrm{~s}$. Meanwhile, assume that each Markov chain $\omega_{i j}$ takes values in a finite state space $S=\{0,1\}$, and the time interval $\Delta=1 \mathrm{~s}$. Otherwise, each undirected edge has a Markov chain, and the six agents have 15 chains totally. The transition rate matrices are considered as Table 2.

As an example, the time evolutions of some Markov chains are depicted in Figure 4.

Let the initial states of six quadrotor UAVs be

$$
\begin{aligned}
& {\left[x_{1}(0), y_{1}(0), z_{1}(0), \phi_{1}(0), \theta_{1}(0), \psi_{1}(0)\right]^{T}} \\
& \quad=[1,1,0,0,0,0]^{T},
\end{aligned}
$$
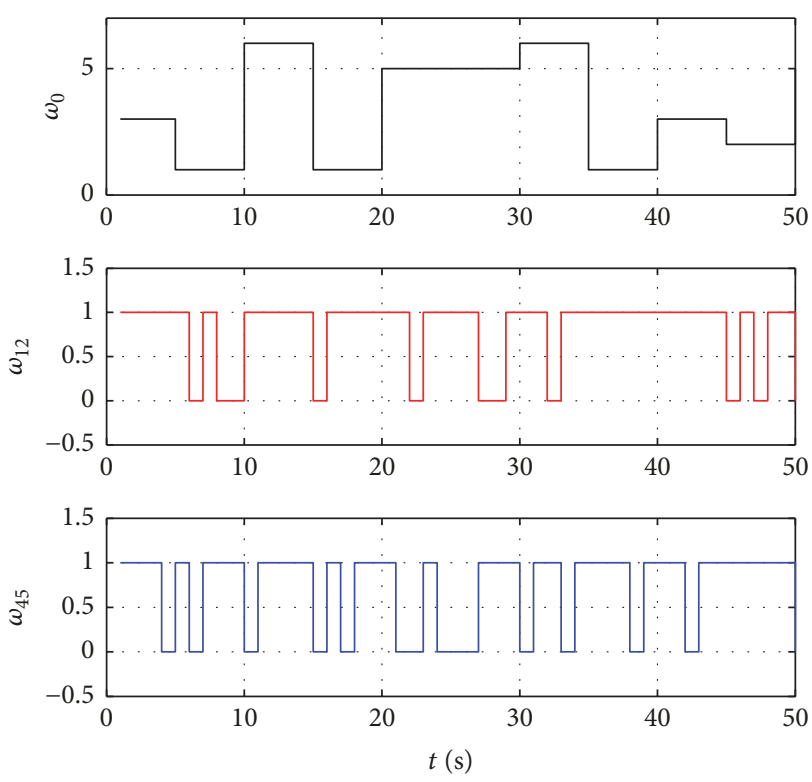

FIGURE 4: Evolutions of some Markov chains.

$$
\begin{aligned}
& \quad\left[x_{2}(0), y_{2}(0), z_{2}(0), \phi_{2}(0), \theta_{2}(0), \psi_{2}(0)\right]^{T} \\
& \quad=[3,-1,0,0,0,0]^{T}, \\
& \quad\left[x_{3}(0), y_{3}(0), z_{3}(0), \phi_{3}(0), \theta_{3}(0), \psi_{3}(0)\right]^{T} \\
& \quad=[-3,2,0,0,0,0]^{T}, \\
& \quad\left[x_{4}(0), y_{4}(0), z_{4}(0), \phi_{4}(0), \theta_{4}(0), \psi_{4}(0)\right]^{T} \\
& \quad=[-2,-2,0,0,0,0]^{T}, \\
& {\left[x_{5}(0), y_{5}(0), z_{5}(0), \phi_{5}(0), \theta_{5}(0), \psi_{5}(0)\right]^{T}} \\
& \quad=[3.5,2,0,0,0,0]^{T}, \\
& \left.\quad x_{6}(0), y_{6}(0), z_{6}(0), \phi_{6}(0), \theta_{6}(0), \psi_{6}(0)\right]^{T} \\
& \quad=[-3,1,0,0,0,0]^{T} .
\end{aligned}
$$

The desired trajectory is given by $R_{d}=[0,0,0.05 t, \pi / 30]^{T}$, and the formation shape specified by the desired interagent distances is described as

$$
\delta_{i}(t)=\left[\begin{array}{c}
\delta_{i x}(t) \\
\delta_{i y}(t) \\
\delta_{i z}(t) \\
\delta_{i \psi}(t)
\end{array}\right]=\left[\begin{array}{c}
3 \sin \left(0.2 t+\frac{(i-1) \pi}{3}\right) \\
3 \cos \left(0.2 t+\frac{(i-1) \pi}{3}\right) \\
0 \\
0
\end{array}\right]
$$

which means that if the quadrotor system achieves the desired time varying formation, then the six quadrotor UAVs will follow a circle while keeping a phase separation of $\pi / 3$. In order to verify the effectiveness and advantage of the 
TABLE 1: The system parameters of quadrotor UAVs.

\begin{tabular}{lccc}
\hline Parameters & Nominal value & Parameters & Nominal value \\
\hline$l_{i}$ & $0.20 \mathrm{~m}$ & $k_{x i}$ & $0.010 \mathrm{~N} / \mathrm{m} \cdot \mathrm{s}^{-1}$ \\
$m_{i}$ & $1.79 \mathrm{~kg}$ & $k_{y i}$ & $0.010 \mathrm{~N} / \mathrm{m} \cdot \mathrm{s}^{-1}$ \\
$I_{x i}$ & $0.03 \mathrm{~kg} \cdot \mathrm{m}^{2}$ & $k_{z i}$ & $0.010 \mathrm{~N} / \mathrm{m} \cdot \mathrm{s}^{-1}$ \\
$I_{y i}$ & $0.03 \mathrm{~kg} \cdot \mathrm{m}^{2}$ & $k_{\phi i}$ & $0.012 \mathrm{~N} / \mathrm{rad} \cdot \mathrm{s}^{-1}$ \\
$I_{z i}$ & $0.04 \mathrm{~kg} \cdot \mathrm{m}^{2}$ & $k_{\theta i}$ & $0.012 \mathrm{~N} / \mathrm{rad} \cdot \mathrm{s}^{-1}$ \\
$g$ & $9.81 \mathrm{~m} / \mathrm{s}^{2}$ & $k_{\psi i}$ & $0.012 \mathrm{~N} / \mathrm{rad} \cdot \mathrm{s}^{-1}$ \\
$K_{\psi i}$ & $0.40 \mathrm{~N} \cdot \mathrm{m}$ & & \\
\hline
\end{tabular}

TABle 2: Transition rate matrices of the Markov chains in graph.

\begin{tabular}{lccc}
\hline Edges & $\left(\begin{array}{c}\left.v_{1}, v_{2}\right) ;\left(v_{2}, v_{4}\right) ;\left(v_{4}, v_{5}\right) \\
\left(v_{3}, v_{5}\right) ;\left(v_{3}, v_{6}\right)\end{array}\right.$ & $\begin{array}{c}\left(v_{1}, v_{3}\right) ;\left(v_{2}, v_{3}\right) ;\left(v_{2}, v_{6}\right) \\
\left(v_{5}, v_{6}\right) ;\left(v_{1}, v_{5}\right)\end{array}$ & $\begin{array}{c}\left(v_{1}, v_{4}\right) ;\left(v_{1}, v_{6}\right) ;\left(v_{2}, v_{5}\right) \\
\left(v_{3}, v_{4}\right) ;\left(v_{4}, v_{6}\right)\end{array}$ \\
\hline $\begin{array}{l}\text { Transition rate } \\
\text { matrices }\end{array}$ & {$\left[\begin{array}{cc}0.2 & 0.8 \\
0.4 & 0.6\end{array}\right]$} & {$\left[\begin{array}{cc}0.5 & 0.5 \\
? & ?\end{array}\right]$} & {$\left[\begin{array}{l}? \\
? \\
?\end{array}\right]$} \\
\hline
\end{tabular}

TABLE 3: Event times of all agents.

\begin{tabular}{ccccccc}
\hline Agent & $v_{1}$ & $v_{2}$ & $v_{3}$ & $v_{4}$ & $v_{5}$ & \\
\hline Case 1 & 131 & 123 & 109 & 126 & 143 & 115 \\
Case 2 & 62 & 48 & 67 & 60 & 61 & 54 \\
\hline
\end{tabular}

developed formation control protocol, the following two cases are evaluated:

Case 1. The distributed event-triggered function (23), (37) and the controller are implemented with the parameters as follows:

$$
\begin{aligned}
\sigma_{0} & =0.6, \\
\sigma_{1} & =0.1, \\
\mu & =0.3, \\
\alpha_{\phi} & =\alpha_{\theta}=\alpha_{\psi}=4.0, \\
\beta_{\phi} & =\beta_{\theta}=\beta_{\psi}=3.2, \\
\alpha_{x} & =\alpha_{y}=\alpha_{z}=2.0, \\
\beta_{x} & =\beta_{y}=\beta_{z}=1.8 .
\end{aligned}
$$

Simulation results are shown in Figures 5 and 6. The response curves of formation trajectory and attitude are given in Figure 5, which reveal that positions of six agents successfully achieve and maintain a predesigned formation shape. As depicted in Figure 6, it demonstrates the snapshots of position trajectories of the six robots at $t=0 \mathrm{~s}, t=5 \mathrm{~s}$, $t=20 \mathrm{~s}$, and $t=40 \mathrm{~s}$, respectively.

Case 2. The same formation task is carried out by implementing the event-triggered controller with a constant threshold parameter $\sigma(t)=\sigma_{0}=0.6$. The aforementioned results derived from theorems indicate that the formation control problem is feasible under the general event-triggered
TABLE 4: Comparison results in Cases 1 and 2.

\begin{tabular}{lcc}
\hline Signals & Case 1 & Case 2 \\
\hline Mean & & \\
$e_{1 \phi}^{i}$ & -0.0281 & -0.0856 \\
$e_{1 \phi}^{i}$ & -0.0257 & -0.0849 \\
$\widetilde{\psi}_{1 i}$ & -0.0319 & -0.1147 \\
$\widetilde{x}_{1 i}$ & 0.0156 & 0.0664 \\
$\tilde{y}_{1 i}$ & 0.0221 & 0.0702 \\
$\widetilde{z}_{1 i}$ & -0.0202 & -0.0445 \\
Standard deviation & & \\
$e_{1 \phi}^{i}$ & 0.1075 & 0.2490 \\
$e_{1 \theta}^{i}$ & 0.1196 & 0.2772 \\
$\widetilde{\psi}_{1 i}$ & 0.1402 & 0.3128 \\
$\widetilde{x}_{1 i}$ & 0.0809 & 0.1824 \\
$\tilde{y}_{1 i}$ & 0.0873 & 0.1905 \\
$\widetilde{z}_{1 i}$ & 0.0894 & 0.1943 \\
\hline
\end{tabular}

controller. Figure 7 shows the trajectories of positions and attitude angles within $50 \mathrm{~s}$ and Figure 8 shows the snapshots of position trajectories at $t=0 \mathrm{~s}, t=5 \mathrm{~s}, t=20 \mathrm{~s}$, and $t=40 \mathrm{~s}$, respectively.

The actual event release instants for all agents are shown in Figures 9 and 10. A quantitative comparison of how many events on each agent are actually transmitted is provided in Table 3, and Table 4 shows the mean and standard deviation for each error signal in $20 \mathrm{~s} \leq t \leq 50 \mathrm{~s}$.

Remark 14. Some conclusions could be summarized as follows: 


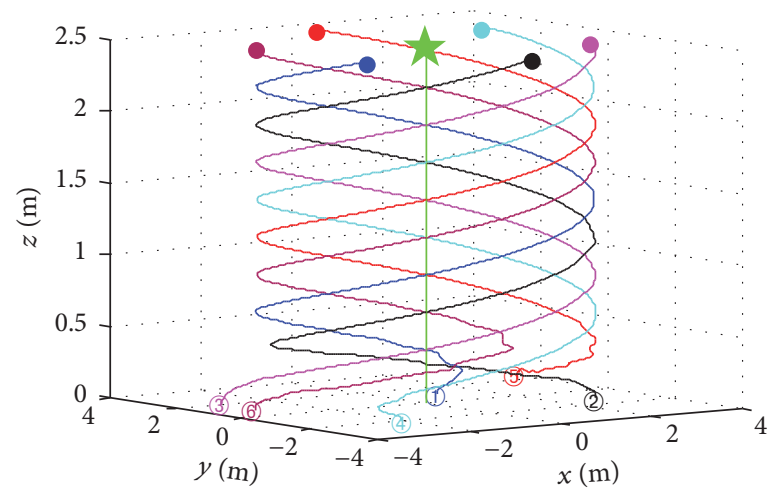

(a) Evolution of the position states
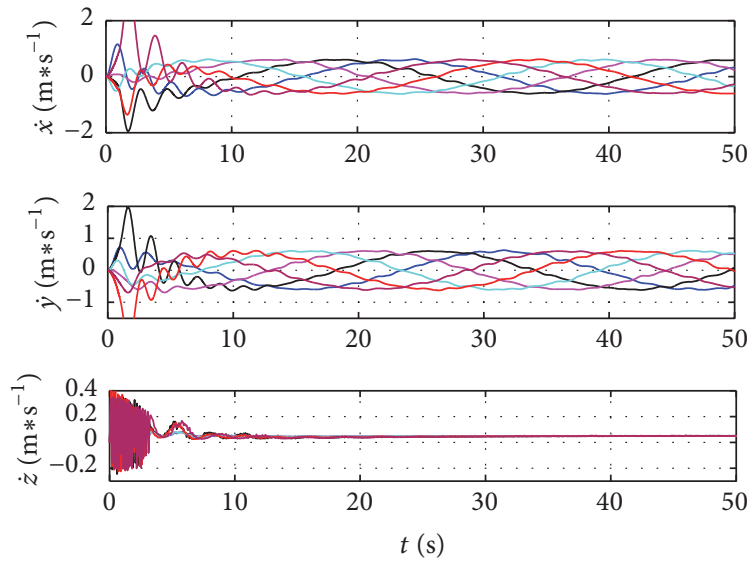

(c) Evolution of the velocity states
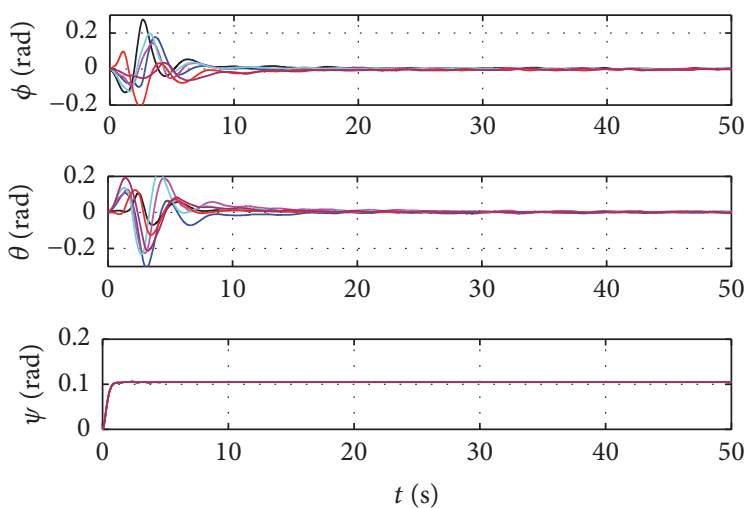

(b) Evolution of the attitude states
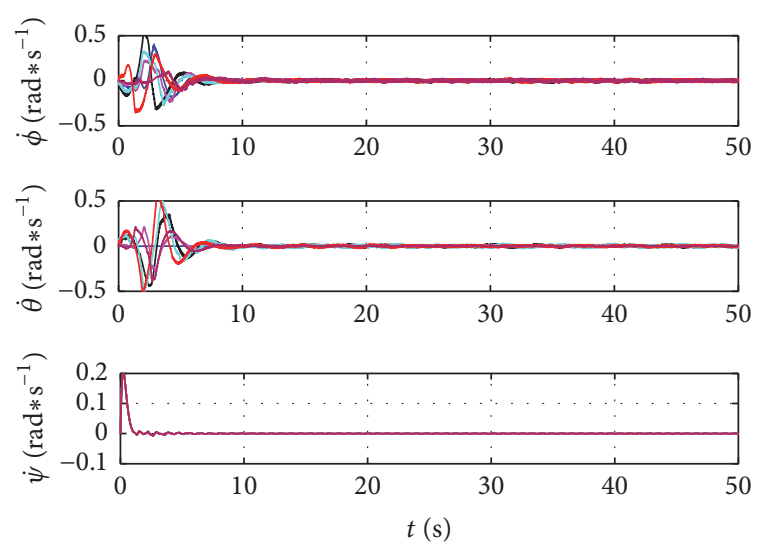

(d) Evolution of the angular velocity

FIgURE 5: Trajectories of the agents within $50 \mathrm{~s}$ in Case 1.
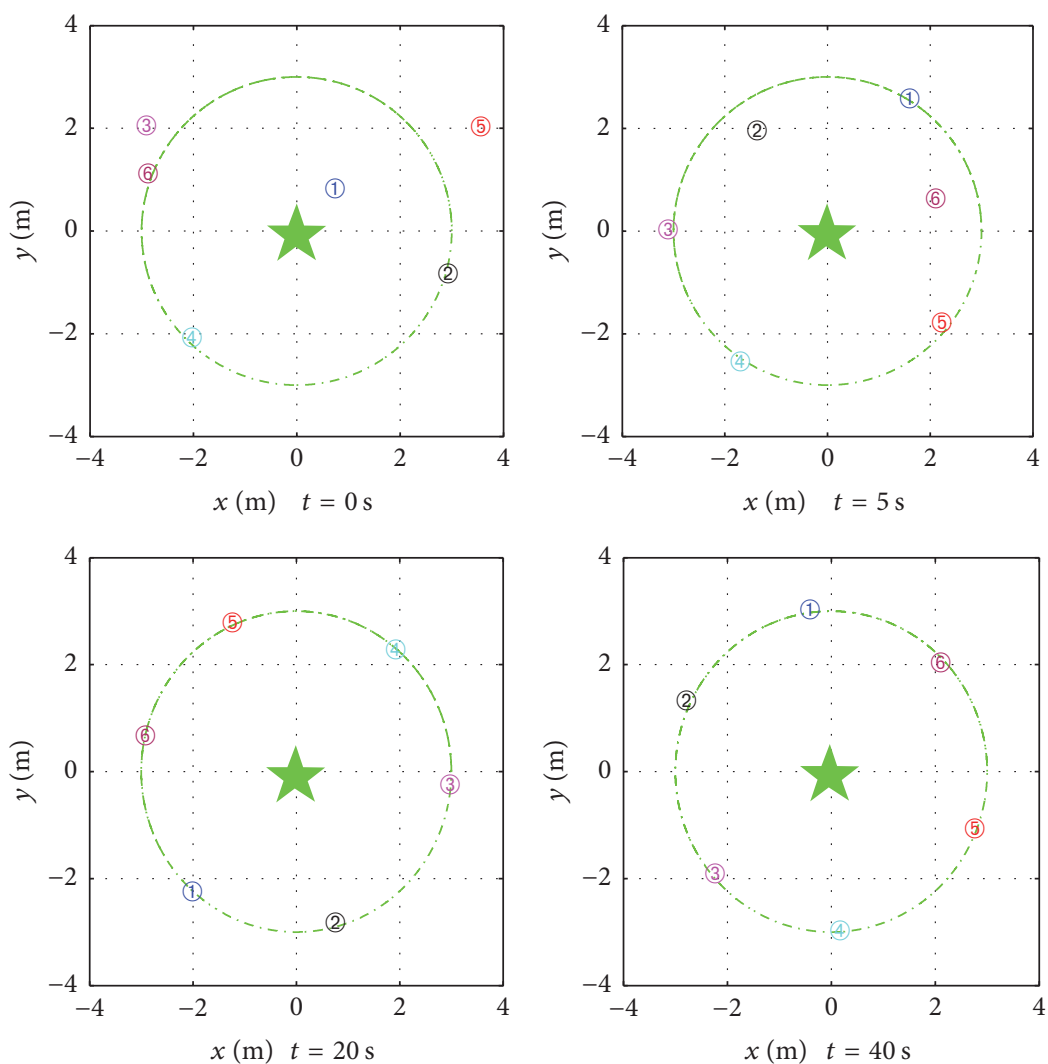

Figure 6: Position snapshots of the six agents in Case 1. 


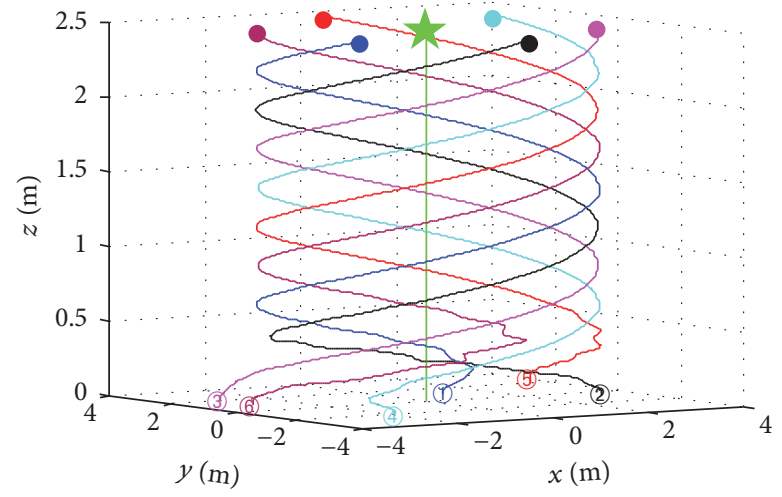

(a) Evolution of the position states
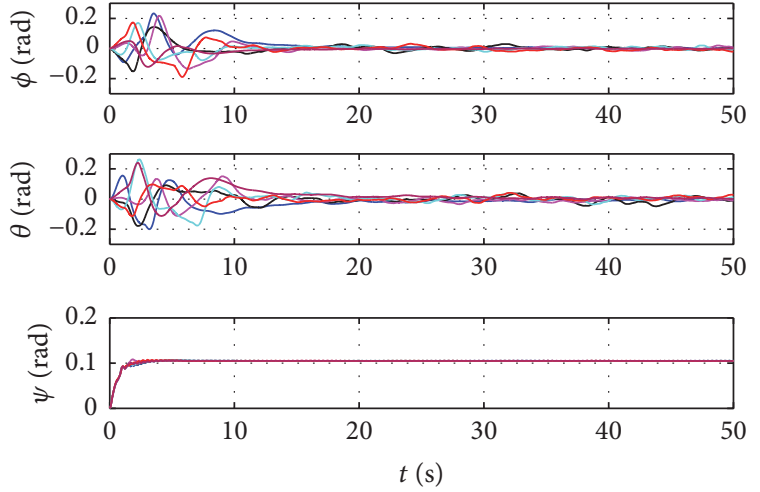

(b) Evolution of the attitude states

FIGURE 7: Trajectories of the agents within $50 \mathrm{~s}$ in Case 2.
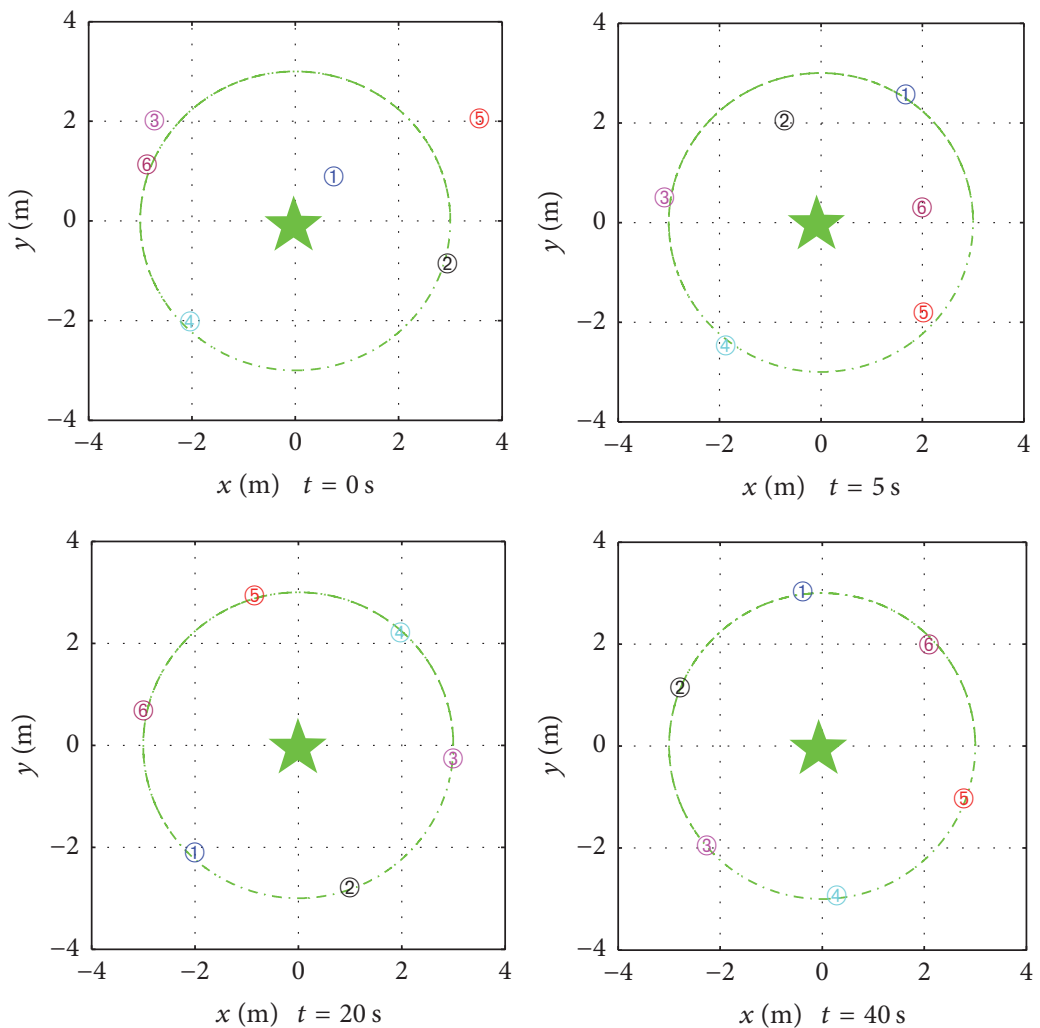

FIgUre 8: Position snapshots of the six agents in Case 2.

(1) Simulation results in both Cases 1 and 2 show that the formation shape could be guaranteed under Markovian switching topologies with partially unknown transition rates.

(2) In Figure 5, the convergence time of $\psi$ and $\dot{\psi}$ (i.e., 0.80 s) is shorter than that of $x, \dot{x}, y, \dot{y}, z$, and $\dot{z}$ (i.e., 7.20 s); these plots have different time horizons; it confirms the twotime-scale structure controller derived from the perturbing parameter $\sigma_{1}$. Particularly, the reason why the convergence time of $\phi$ and $\theta$ in Figure 5 is as long as that of the position states is that $\phi$ and $\theta$ are tracking the reference $\phi_{i d}$ and $\theta_{i d}$ rather than zero.
(3) As shown in Figures 6 and 8 , at $t=0 \mathrm{~s}, t=20 \mathrm{~s}$, and $t=$ $40 \mathrm{~s}$, the real shape of six agents is always uniform. When $t=$ $5 \mathrm{~s}$, there is a little difference because the graph is randomly governed by Markov chains.

(4) Compared with Case 2, there are more events for all agents in Case 1, which means that it has more potential to reduce the occupancy of limited communication resources in Case 2. That is because the threshold parameter $\sigma(t)$ is fixed as $\sigma_{0}$ all the time, while in Case $1, \sigma(t)$ is monotonically nonincreasing (shown in Figure 11). Generally, the larger the value of the threshold parameter is, the less events are derived. 


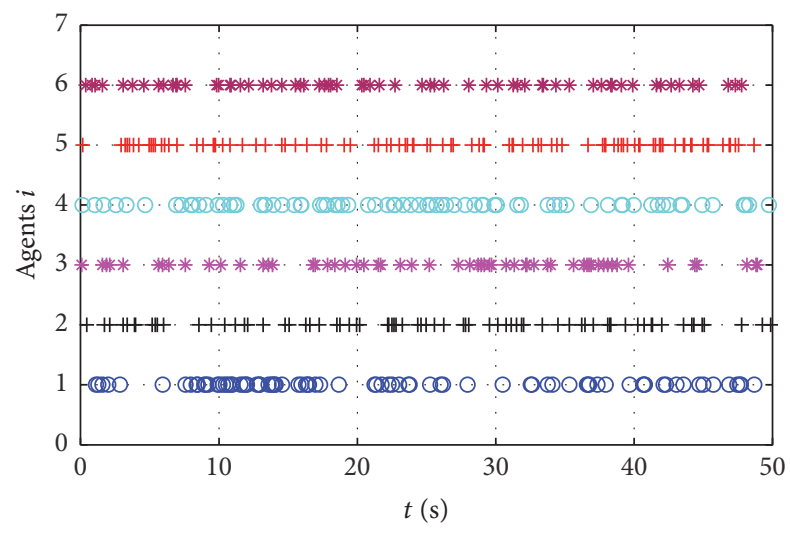

Figure 9: Events times of all agents in Case 1.

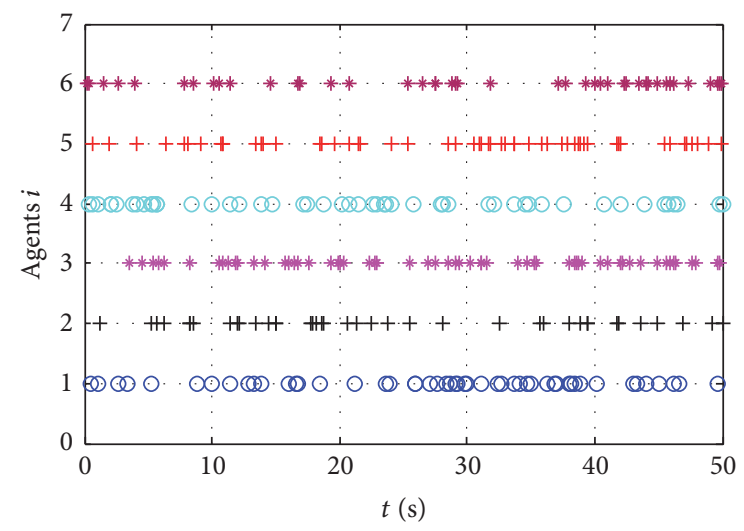

Figure 10: Events times of all agents in Case 2.

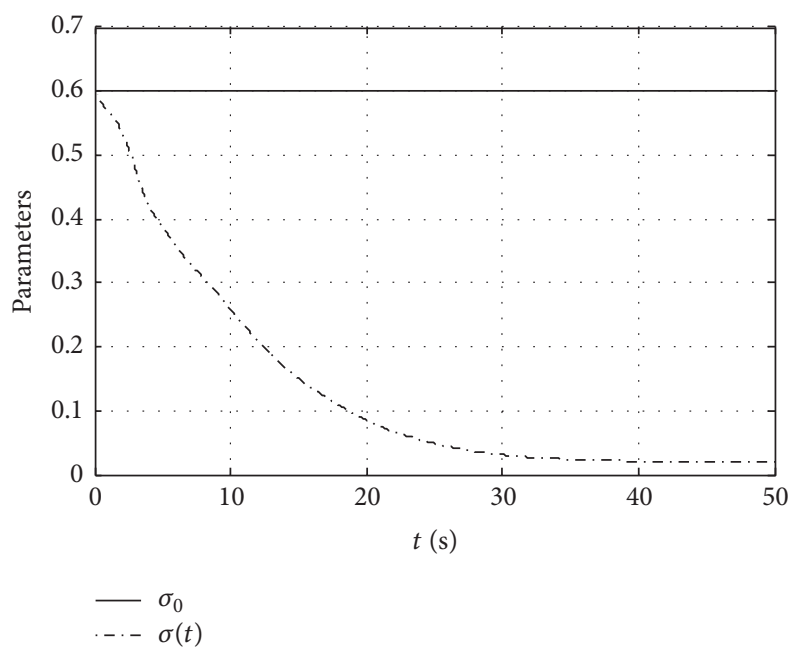

FIgURE 11: The threshold parameters in Cases 1 and 2.

(5) Based on most of the computed performance indexes in Table 4, the controller in Case 1 can achieve better formation performance than that in Case 2. Although less events are derived by using a fixed threshold parameter, the formation performance is compromised.

\section{Conclusion}

In this paper, formation control problem of multiple quadrotor UAVs under Markovian switching topologies is addressed through an event-triggered control strategy. Markovian switching topologies are redesigned through utilizing the Markov chains to the edge set, which could recover the traditional ones by adjusting the modes of edges and the transition rates. Then, a predesigned formation shape can be kept along with a distributed formation controller constructed with a reasonably event-triggered updated rule, even subject to the unknown transition rates. Rigorous analyses of the convergence results are obtained based on singular perturbations theory and Lyapunov stability theory. In addition, Zeno behavior is excluded for the triggered time sequences. Simulation results have been given to illustrate the effectiveness of the proposed control strategy. In some of the cases, the velocity states are unmeasurable. Since that, event-triggered formation control problem for multiple quadrotor UAVs without collision subject to output feedback will be considered in our future work.

\section{Conflicts of Interest}

The authors declare that there are no conflicts of interest regarding the publication of this paper.

\section{Acknowledgments}

This work is supported by NSFC under Grant 61473248 and Natural Science Foundation of Hebei Province under Grant F2016203496.

\section{References}

[1] J. Yu, J. Ji, Z. Miao, and J. Zhou, "Adaptive formation control of networked Lagrangian systems with a moving leader," Nonlinear Dynamics, pp. 1-12, 2017.

[2] K.-K. Oh, M.-C. Park, and H.-S. Ahn, "A survey of multi-agent formation control," Automatica, vol. 53, pp. 424-440, 2015.

[3] A. Franchi, C. Secchi, M. Ryll, H. Bülthoff, and P. R. Giordano, "Shared control: Balancing autonomy and human assistance with a group of quadrotor UAVs," IEEE Robotics and Automation Magazine, vol. 19, no. 3, pp. 57-68, 2012.

[4] W. Hao and B. Xian, "Nonlinear adaptive fault-tolerant control for a quadrotor UAV based on immersion and invariance methodology," Nonlinear Dynamics, pp. 1-14, 2017.

[5] A. Abdessameud and A. Tayebi, "Formation control of VTOL Unmanned Aerial Vehicles with communication delays," Automatica, vol. 47, no. 11, pp. 2383-2394, 2011.

[6] H. Qiu and H. Duan, "Receding horizon control for multiple UAV formation flight based on modified brain storm optimization," Nonlinear Dynamics, vol. 78, no. 3, pp. 1973-1988, 2014.

[7] Y.-Y. Chen, Z.-Z. Wang, Y. Zhang, C.-L. Liu, and Q. Wang, "A geometric extension design for spherical formation tracking control of second-order agents in unknown spatiotemporal flowfields," Nonlinear Dynamics, vol. 88, no. 2, pp. 1173-1186, 2017.

[8] Z. Hou, W. Wang, G. Zhang, and C. Han, "A survey on the formation control of multiple quadrotors," in Proceedings of the 2017 14th International Conference on Ubiquitous Robots and 
Ambient Intelligence (URAI), pp. 219-225, IEEE, Jeju, South Korea, 2017.

[9] Y. Wang, Q. Wu, and Y. Wang, "Distributed cooperative control for multiple quadrotor systems via dynamic surface control," Nonlinear Dynamics, vol. 75, no. 3, pp. 513-527, 2014.

[10] Y. Xia, X. Na, Z. Sun, and J. Chen, "Formation control and collision avoidance for multi-agent systems based on position estimation," ISA Transactions, vol. 61, pp. 287-296, 2016.

[11] J. Ghommam, L. F. Luque-Vega, B. Castillo-Toledo, and M. Saad, "Three-dimensional distributed tracking control for multiple quadrotor helicopters," Journal of The Franklin Institute, vol. 353, no. 10, pp. 2344-2372, 2016.

[12] H. Du, W. Zhu, G. Wen, and D. Wu, "Finite-time formation control for a group of quadrotor aircraft," Aerospace Science and Technology, vol. 69, pp. 609-616, 2017.

[13] X. Dong, L. Han, Q. Li, and Z. Ren, "Time-varying formation control for double-integrator multi-agent systems with jointly connected topologies," International Journal of Systems Science, vol. 47, no. 16, pp. 3829-3838, 2016.

[14] X. Dong, Y. Zhou, Z. Ren, and Y. Zhong, "Time-Varying Formation Tracking for Second-Order Multi-Agent Systems Subjected to Switching Topologies With Application to Quadrotor Formation Flying," IEEE Transactions on Industrial Electronics, vol. 64, no. 6, pp. 5014-5024, 2017.

[15] F. Liao, R. Teo, J. L. Wang, X. Dong, F. Lin, and K. Peng, "Distributed Formation and Reconfiguration Control of VTOL UAVs," IEEE Transactions on Control Systems Technology, vol. 25, no. 1, pp. 270-277, 2017.

[16] X. Dong and G. Hu, "Time-varying formation control for general linear multi-agent systems with switching directed topologies," Automatica, vol. 73, pp. 47-55, 2016.

[17] Y. Shang and Y. Ye, "Leader-follower fixed-time group consensus control of multiagent systems under directed topology," Complexity, vol. 2017, Article ID 3465076, 9 pages, 2017.

[18] M. Faraji-Niri, M. R. Jahed-Motlagh, and M. BarkhordariYazdi, "Stabilization of active fault-tolerant control systems by uncertain nonhomogeneous Markovian jump models," Complexity, vol. 21, no. S1, pp. 318-329, 2016.

[19] X. Ge and Q.-L. Han, "Consensus of Multiagent Systems Subject to Partially Accessible and Overlapping Markovian Network Topologies," IEEE Transactions on Cybernetics, vol. 47, no. 8, pp. 1807-1819, 2017.

[20] A. Wang, T. Dong, and X. Liao, "Event-triggered synchronization strategy for complex dynamical networks with the Markovian switching topologies," Neural Networks, vol. 74, pp. 52-57, 2016.

[21] L. Rong and H. Shen, "Distributed containment control of second-order multiagent systems with input delays under general protocols," Complexity, vol. 21, no. 6, pp. 112-120, 2016.

[22] C. Viel, S. Bertrand, M. Kieffer, and H. Piet-Lahanier, "Distributed event-triggered control for multi-agent formation stabilization," IFAC-PapersOnLine, vol. 50, no. 1, pp. 8025-8030, 2017.

[23] X.-M. Zhang and Q.-L. Han, "Event-triggered dynamic output feedback control for networked control systems," IET Control Theory \& Applications, vol. 8, no. 4, pp. 226-234, 2014.

[24] X. Ge and Q.-L. Han, "Distributed Formation Control of Networked Multi-Agent Systems Using a Dynamic Event-Triggered Communication Mechanism," IEEE Transactions on Industrial Electronics, vol. 64, no. 10, pp. 8118-8127, 2017.
[25] X. Yi, J. Wei, D. V. Dimarogonas, and K. H. Johansson, Formation control for multi-agent systems with connectivity preservation and event-triggered controllers, https://arxiv.org/abs/ 1611.03105v4, 2016.

[26] X. Ge, Q.-L. Han, and F. Yang, "Event-Based Set-Membership Leader-Following Consensus of Networked Multi-Agent Systems Subject to Limited Communication Resources and Unknown-But-Bounded Noise," IEEE Transactions on Industrial Electronics, vol. 64, no. 6, pp. 5045-5054, 2017.

[27] C. Peng, Y. Song, P. Xie X et al., "Event-triggered output tracking control for wireless networked control systems with communication delays and data dropouts," IET Control Theory \& Applications, vol. 10, no. 17, pp. 2195-2203, 2016.

[28] H. Zhang, R. Yang, H. Yan, and F. Yang, " $H_{\infty}$ consensus of event-based multi-agent systems with switching topology," Information Sciences, vol. 370, pp. 623-635, 2016.

[29] C. Peng, J. Zhang, and Y. C. Yan, "Adaptive event-triggering $H_{\infty}$ load frequency control for network-based power systems," IEEE Transactions on Industrial Electronics, vol. 65, no. 2, pp. 16851694, 2018.

[30] J. Lee, R. Mukherjee, and H. K. Khalil, "Output feedback stabilization of inverted pendulum on a cart in the presence of uncertainties," Automatica, vol. 54, pp. 146-157, 2015.

[31] M. T. Ravichandran and A. D. Mahindrakar, "Robust stabilization of a class of underactuated mechanical systems using time scaling and Lyapunov redesign," IEEE Transactions on Industrial Electronics, vol. 58, no. 9, pp. 4299-4313, 2011.

[32] S. González-Vázquez and J. Moreno-Valenzuela, "Motion control of a quadrotor aircraft via singular perturbations," International Journal of Advanced Robotic Systems, vol. 10, 2013.

[33] J. Zhou, H. Dong, and J. Feng, "Event-triggered communication for synchronization of Markovian jump delayed complex networks with partially unknown transition rates," Applied Mathematics and Computation, vol. 293, pp. 617-629, 2017.

[34] D. Xie, S. Xu, Z. Li, and Y. Zou, "Event-triggered consensus control for second-order multi-agent systems," IET Control Theory \& Applications, vol. 9, no. 5, pp. 667-680, 2015.

[35] K. Vladimír, "A Contribution to Matrix Quadratic Equations," IEEE Transactions on Automatic Control, vol. 17, no. 3, pp. 344$347,1972$. 


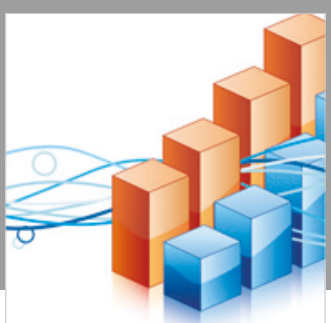

Advances in

Operations Research

\section{-n-m}
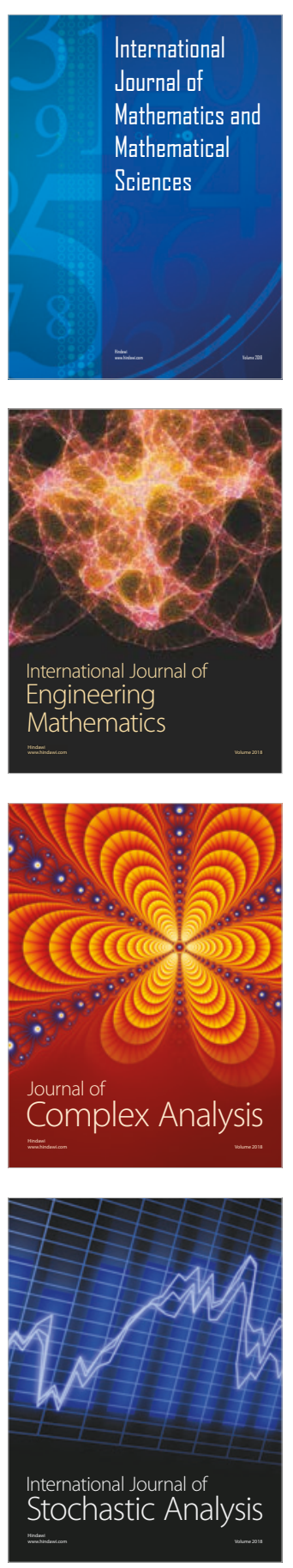
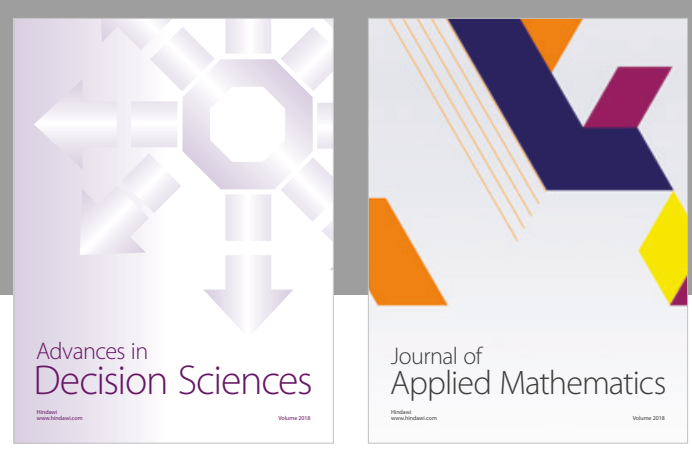

Journal of

Applied Mathematics
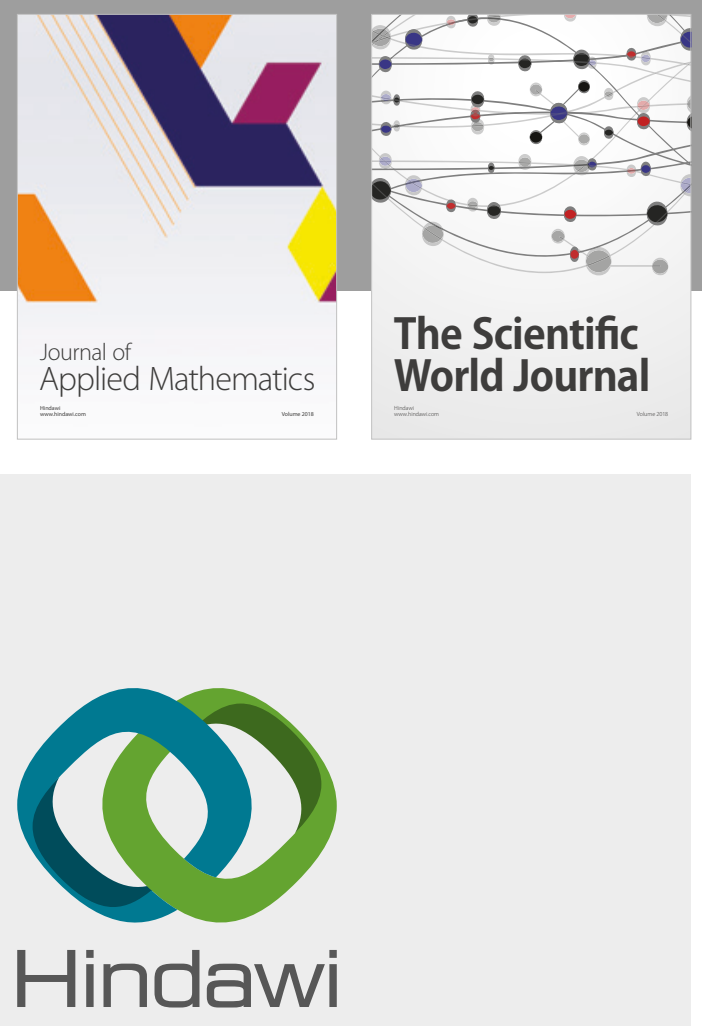

Submit your manuscripts at

www.hindawi.com

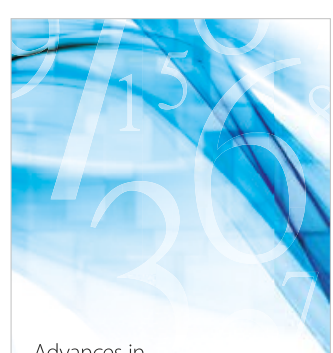

Advances in
Numerical Analysis
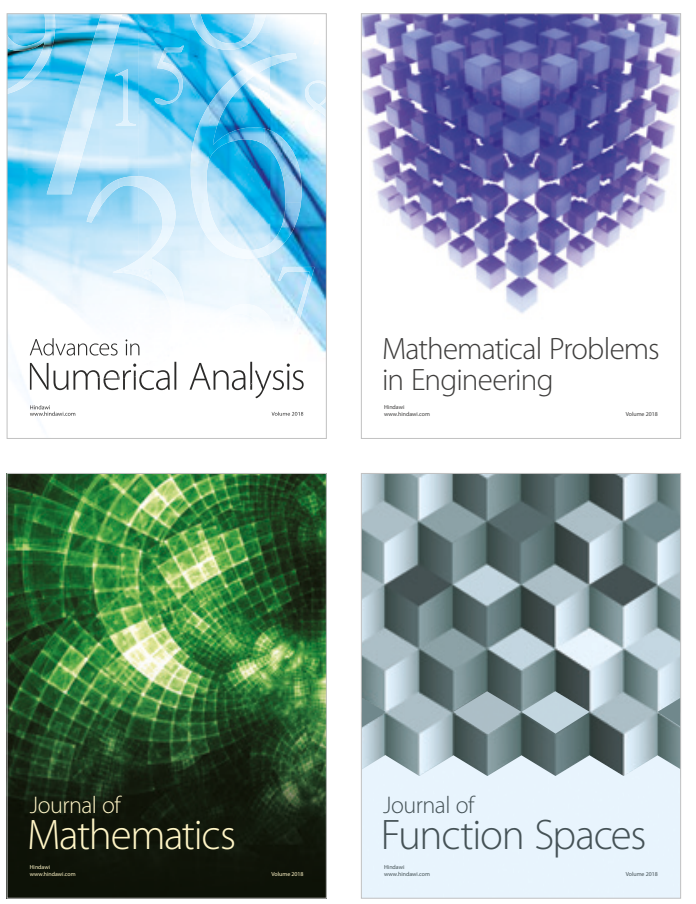

Mathematical Problems in Engineering

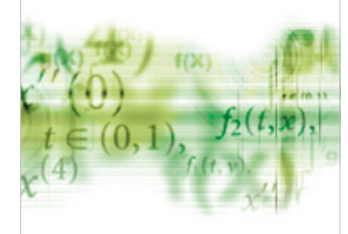

International Journal of

Differential Equations

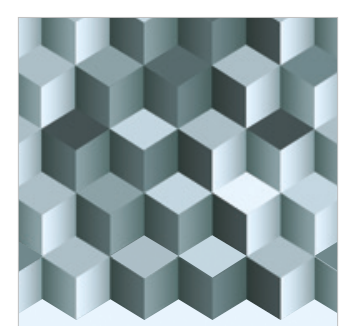

Journal of

Function Spaces
The Scientific

World Journal

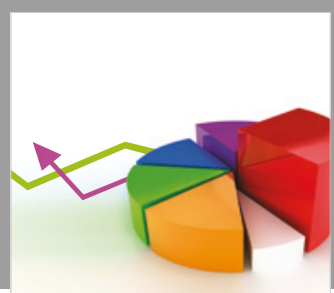

Journal of

Probability and Statistics
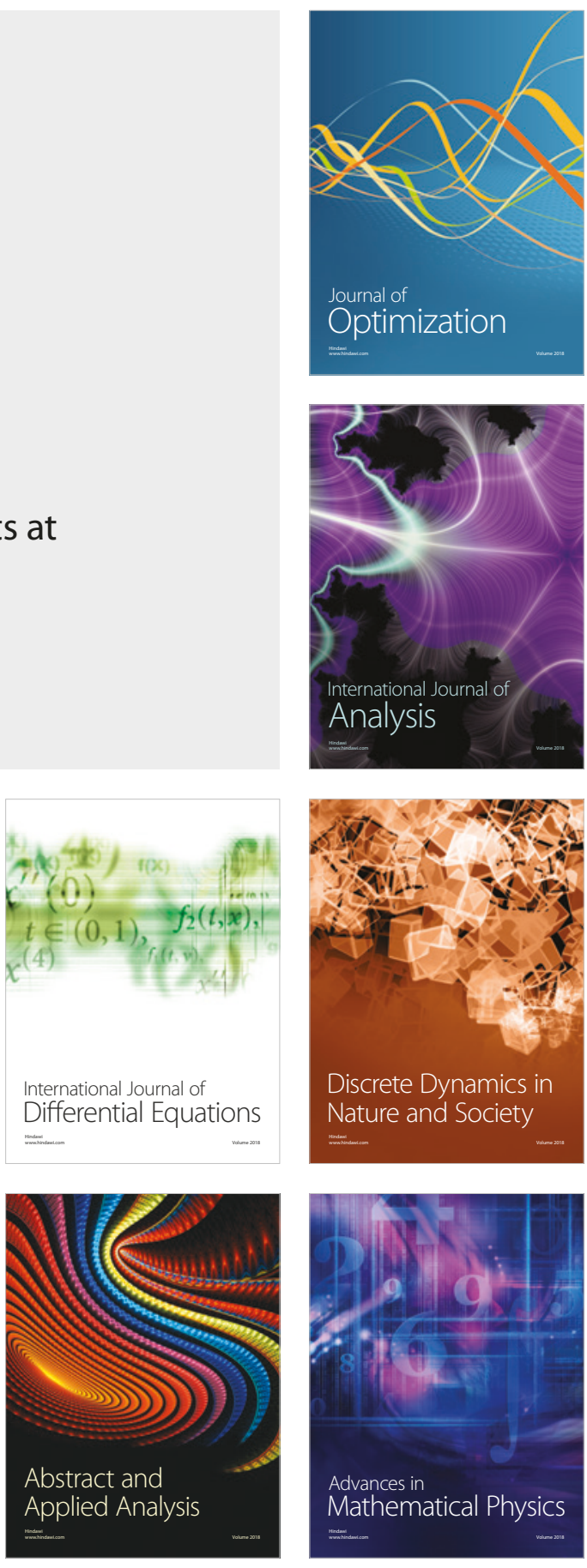